Document downloaded from:

http://hdl.handle.net/10251/47648

This paper must be cited as:

Coupé, P.; Manjón Herrera, JV.; Chamberland, M.; Descoteaux, M.; Hiba, B. (2013). Collaborative patch-based super-resolution for diffusion-weighted images. Neurolmage. 83:245-261. doi:10.1016/j.neuroimage.2013.06.030

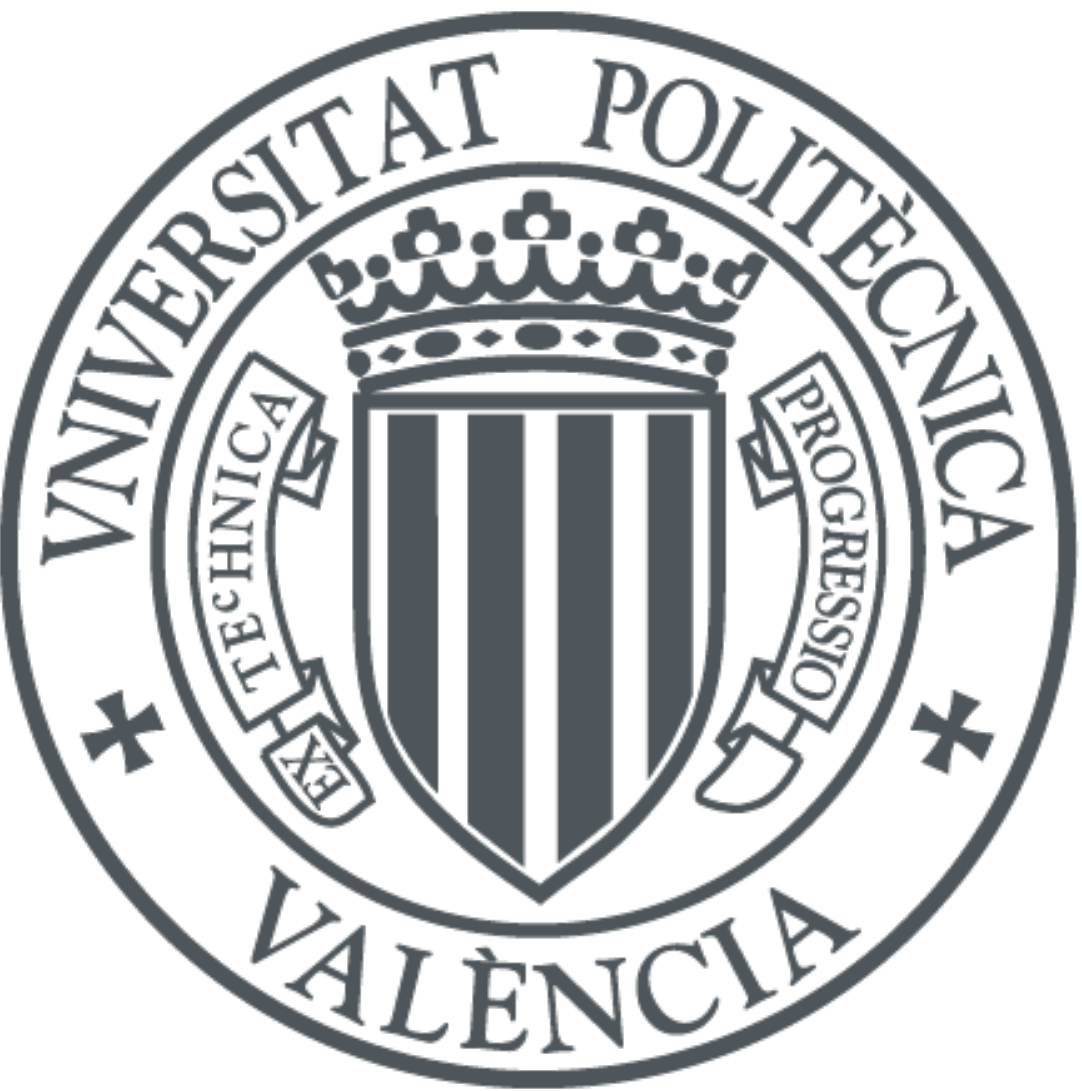

The final publication is available at

http://dx.doi.org/10.1016/j.neuroimage.2013.06.030

Copyright Elsevier

Additional Information 


\title{
Collaborative Patch-Based Super-Resolution for Diffusion-Weighted Images
}

\author{
Pierrick Coupé ${ }^{1}$, José V. Manjón ${ }^{2}$, Maxime Chamberland ${ }^{3}$, Maxime Descoteaux $^{3}$, Bassem Hiba ${ }^{4}$ \\ * Corresponding author: pierrick.coupe@gmail.com \\ ${ }^{1}$ Laboratoire Bordelais de Recherche en Informatique, Unité Mixte de Recherche CNRS (UMR 5800), Bordeaux, France \\ ${ }^{2}$ Instituto de Aplicaciones de las Tecnologías de la Información y de las Comunicaciones Avanzadas (ITACA), \\ Universidad Politécnica de Valencia, Camino de Vera s/n, 46022 Valencia, Spain \\ ${ }^{3}$ Sherbrooke Connectivity Imaging Lab (SCIL), Département d'Informatique Université de Sherbrooke, 2500 Boulevard \\ de l'Université, Sherbrooke, QC, J1K2R1, Canada \\ ${ }^{4}$ Centre de Résonance Magnétique des Systèmes Biologiques, UMR 5536 CNRS, Université Bordeaux Segalen, F- \\ 33076 Bordeaux, France
}

\begin{abstract}
In this paper, a new single image acquisition super-resolution method is proposed to increase image resolution of diffusion weighted (DW) images. Based on a nonlocal patch-based strategy, the proposed method uses a non diffusion image (b0) to constrain the reconstruction of DW images. An extensive validation is presented with a gold standard built on averaging 10 high-resolution DW acquisitions. A comparison with classical interpolation methods such as trilinear and B-spline demonstrates the competitive results of our proposed approach in terms of improvements on image reconstruction, fractional anisotropy (FA) estimation, generalized FA and angular reconstruction for tensor and high angular resolution diffusion imaging (HARDI) models. Besides, first results of reconstructed ultra high resolution DW images are presented at $0.6 \times 0.6 \times 0.6 \mathrm{~mm}^{3}$ and $0.4 \times 0.4 \times 0.4 \mathrm{~mm}^{3}$ using our gold standard based on the average of 10 acquisitions, and on a single acquisition. Finally, fiber tracking results show the potential of the proposed super-resolution approach to accurately analyze white matter brain architecture.
\end{abstract}

Keywords: super-resolution, nonlocal means, patch-based method, diffusionweighted imaging (DWI), diffusion tensor imaging (DTI), high angular resolution diffusion imaging (HARDI), ultra high resolution DWI/DTI/HARDI. 


\section{Introduction}

Diffusion weighted (DW) imaging enables the reconstruction of information revealing the shape, the coherence and the integrity of brain tissues microstructure which can be indirectly analyzed through the assessment of motion of water molecules. This technique has been applied widely for in-vivo analysis of white matter architecture (Assaf, 2008; Basser et al., 1994; Jbabdi et al., 2010) and has recently been applied to the study of gray matter (Sagi et al., 2012). Moreover, Diffusion Tensor Imaging (DTI) has been used to study a large range of neurological diseases such as Alzheimer's dementia (Huang et al., 2007) and Parkinson's disease (Gattellaro et al., 2009), or to quantify other causes of tissue degradations such as cancer (Price et al., 2003) and aging (Hsu et al., 2010). Despite its interesting properties, DW imaging is an inherently low signal-to-noise ratio (SNR) technique and yields to relatively poor spatial resolution. In clinical conditions, the DWI is characterized by a low SNR due to the diffusion-weighting of MR signal, the use of EPI scanning of Fourier space and the T2 decay.

It has been shown that the noise corrupting DW images introduces a bias in diffusion parameter estimation (Basser et al., 1994) and that the partial volume effect (PVE) due to limited spatial resolution introduces a bias in DW imaging analysis (Alexander et al., 2001; Oouchi et al., 2007). Therefore, to improve the sensitivity of studies based on DW imaging, the use of high resolution $(\mathrm{HR})$ or ultrahigh resolution (UHR) DW images with high SNR has to be considered. Such HR and UHR DW images with high SNR could provide a better sensitivity for the study of brain microstructure (Mori and van Zijl, 2002; Mukherjee et al., 2008; Zeineh et al., 2012). Moreover, these images could play an important role in fiber tractography to enable tracking of finer bundles or to better handle the fiber crossing problem (Dyrby et al., 2011; Miller et al., 2011; Raffelt et al., 2012). However, the acquisition of such HR or UHR images remains a challenging problem in clinical conditions since improvement in resolution is obtained at the cost of either lower SNR, longer acquisition time or both. Indeed, to increase resolution at the acquisition stage, higher frequencies in k-space are sampled. In addition to increase the acquisition time, the reduced voxel size results in lower SNR and thus usually requires the averaging of several acquisitions that will even more increase the acquisition time. Therefore, such approaches are limited in clinical applications. For instance, five days of scanning followed by the averaging of five acquisitions were required in (Miller et al., 2011) to obtain post-mortem UHR DW images with high SNR at $0.73 \times 0.73 \times 0.73 \mathrm{~mm}^{3}$. To limit acquisition time, less efficient approaches can be used to obtain UHR DW images such as zero padding in k-space. For instance, one hour was required to obtain and average seven acquisitions in (Zeineh et al., 2012) to construct HR DW images at $1.4 \times 1.4 \times 1.4 \mathrm{~mm}^{3}$ reconstructed at $0.7 \times 0.7 \times 0.7 \mathrm{~mm} 3$ using zero-padding. However zero-padding is similar to performing interpolation in image space and is known to introduce blur and diminish contrast (Van Reeth et al., 2012).

To enable acquisition of HR images without long acquisition times, super-resolution (SR) acquisition techniques have been investigated in the past (initially proposed for MRI and more recently for DWI). Such methods are based on the combination of multiple low resolution (LR) images to reconstruct a HR image. Methods using sub-pixel shifting strategies have been proposed in the in-plane dimension (Peled and Yeshurun, 2001). It was however shown by (Greenspan et al., 2002) that it does not provide any resolution enhancement because in-plane shifting is equivalent to a global phase shift in k-space. Therefore, Greenspan et al. (2002) suggested to perform subpixel shifting in the slice direction.

Another possible strategy consists in fusing several anisotropic acquisitions with a high in-plane resolution only along one axis. Inspired by works on fetal imaging (Clouchoux et al., 2010; Gholipour et al., 2010; Rousseau et al., 2006), the fusion of 3 orthogonal acquisitions has been recently applied to DW images (Scherrer et al., 2012). This strategy was then extended to arbitrary 
orientations (Poot et al., 2010) and applied to DW images (Poot et al., 2012). Reviews and comparisons of some of these methods can be found in (Plenge et al., 2012; Van Reeth et al., 2012).

In contrast to SR acquisition techniques that require specific acquisition protocols of multiple LR images, there exists also a category of single image SR methods. While in the literature the term super-resolution is used for both categories, they derived from different paradigms since single image SR techniques are pure post-processing methods and thus are totally independent from the acquisition protocol. Therefore, these methods can be combined with SR acquisition techniques based on multiple LR images. A review of single image SR techniques for natural images can be found in (van Ouwerkerk, 2006). Recently such post-processing, single image, super-resolution methods have been proposed for MRI (Manjon et al., 2010a; Manjon et al., 2010b; Rousseau, 2008; Rousseau, 2010). The main idea is to use the image content to reconstruct information at higher-resolution. To the best of our knowledge, such approaches have not yet been applied to DW images.

Finally, in a different way, a new concept of the SR problem for DW imaging has been proposed in (Calamante et al., 2012a; Calamante et al., 2010; Calamante et al., 2012b; Nedjati-Gilani et al., 2008). Instead of increasing image resolution, the authors proposed to reconstruct a HR density map by using the extra information resulting from tractography. By using neighborhood information contained in the streamlines, intra-voxel density can be estimated with higher spatial resolution than that of the source DW image to obtain super-resolution track-density images. However, this type of method is built on approaches that are similar to tractography methods and thus do not reconstruct high frequency content of images (Scherrer et al., 2012).

In this paper, we investigate the possibility to increase the spatial resolution of DW images using single image SR methods. By applying this type of image post-processing approach on classical DW images (i.e., with voxel sizes typically around $2 \times 2 \times 2 \mathrm{~mm}^{3}$ ), HR DW images can be obtained directly. Moreover, by using HR DW image acquisition, ultra-high resolution DW image can be reconstructed. In this study, we first validate the reconstruction of $1.2 \times 1.2 \times 1.2 \mathrm{~mm}^{3} \mathrm{DW}$ images from $2.4 \times .2 .4 \times 2.4 \mathrm{~mm}^{3} \mathrm{DW}$ images using a HR DWI gold standard (at $1.2 \times 1.2 \times 1.2 \mathrm{~mm}^{3}$ ). Then, we investigate the possibility to reconstruct UHR DW images by applying the proposed SR method on our HR gold standard obtained by averaging several acquisitions to improve SNR. Finally, preliminary results of UHR DW images obtained in a compatible clinical time (i.e., using one acquisition acquired in less than 6 minutes) are presented. The advantages and limitations of our method are finally discussed in the last section. 


\section{Materials and Methods}

\subsection{Patch-Based Super-resolution}

In this paper, we propose a new method that directly increases the spatial resolution of the image by reconstructing high frequency information using the self-similarity property of images. The selfsimilarity property of images has been first investigated for image denoising purposes (Buades et al., 2005). Such an approach considers that two pixels with similar neighborhoods can be viewed as two realizations of the same process. By averaging a large number of pixels considered as belonging to similar realizations, the original process can be accurately reconstructed. The recognized efficiency of this type of nonlocal patch-based approach is mainly due to the high degree of pattern redundancy present in natural images. This fact enables the extraction of many realizations of a given process from the image itself. This self-similarity property has been recently verified for anatomical MRI (Coupe et al., 2008; Manjon et al., 2008) and DWI (Descoteaux et al., 2008; Wiest-Daessle et al., 2008) for denoising tasks.

Such redundancy-based or self-similarity-based priors on image content can be also applied to many other purposes such as super-resolution. In fact, the nonlocal patch-based framework is particularly well-suited to SR since the multiple image acquisitions can be replaced by patch comparison within the image itself to find multiple realizations of the same process. Therefore, Patch-Based Super-Resolution methods (PBSR) have been proposed successfully for natural images (Kindermann et al., 2005; Lou et al., 2010; Mignotte, 2008; Peyré et al., 2008; Protter et al., 2009; Yang et al., 2010b).

Recently, PBSR methods have been introduced and validated for MRI (Manjon et al., 2010a; Manjon et al., 2010b; Rousseau, 2008; Rousseau, 2010). PBSR was first proposed for multimodal reconstruction by Rousseau in (2008). The main idea is to use a HR image to drive the reconstruction of a LR image from another modality (e.g., using a HR T1-w to reconstruct a LR T2w at HR). More recently, this type of approach was extended using a model of the physical acquisition process to improve reconstruction quality (Manjon et al., 2010a). In addition, the possibility to reconstruct HR MRI using only the LR image itself has been investigated for MRI super-resolution (Manjon et al., 2010b; Rousseau, 2010). In this case, only the self-similarity prior is used to drive the reconstruction procedure. Contrary to classical interpolation approaches (linear, cubic, B-spline, ...), which result in blurred reconstructions, the nonlocal patch-based strategy can reconstruct high frequency information by enforcing pattern regularity rather than imposing some smoothness constraint, thus yielding better image quality reconstructions. Since its introduction, this type of prior has demonstrated very high performance compared to classical interpolation methods for MRI.

In this paper, we propose to extend and to adapt the PBSR method to DW datasets. First, we propose a locally adaptive optimization scheme in order to improve the reconstruction quality. Based on a coarse to fine optimization scheme, we use the local image context to drive the reconstruction process. Second, we propose a collaborative approach where the $b 0$ image is used to constrain DW image reconstruction. To do that, the b0 image is reconstructed first to higher resolution using PBSR. Then, each DW image is reconstructed to higher resolution using multimodal PBSR. The contributions of this work are: i) the introduction of a new locally adaptive strategy to improve image reconstruction, ii) the proposition of an original collaborative framework, iii) an extended validation on clinical DW images, iv) the investigation of the impact of our superresolution method on diffusion parameters and tensor or high angular resolution diffusion imaging (HARDI) reconstructions and $v$ ) the presentation of our first results of UHR DW images with fiber tracking examples. 
First, let us define the generic model that describes the relationship between a LR volume $y$ and its corresponding unknown high-resolution (HR) volume $x$ :

$$
y=D H x+\eta
$$

where $D$ is the decimator operator, $H$ is the expected degradation model between $x$ and $y$, and $\eta$ is the acquisition noise. In the following, the operator $H$ is approximated by a 3D boxcar function as done in (Bai et al., 2004; Carmi et al., 2006; Manjon et al., 2010a; Manjon et al., 2010b; Rousseau, 2010).

The super-resolution process consists in reconstructing the HR image $x$ from LR observation $y$. By using classical smoothness constraint on the reconstructed image, the SR reconstruction problem can be written as:

$$
\hat{x}=\arg \min _{x}\left\{|y-D H x|^{2}+\gamma \Phi(x)\right\}
$$

$\Phi(x)$ is the regularization term containing our prior on the image content and $\gamma$ is the regularization parameter controlling the balance between the fidelity term and the regularization term. As shown in (Manjon et al., 2010a; Manjon et al., 2010b; Rousseau, 2008; Rousseau, 2010), an efficient way to define the regularization term is to use nonlocal patch-based approach:

$$
\Phi(x)=\sum_{i \in \Omega}\left\|x_{i}-\psi\left(x_{i}\right)\right\|^{2}
$$

where $\Omega$ is the image grid of $x$ and $\psi$ is the nonlocal means estimator defined as follows:

$$
\psi\left(x_{i}\right)=\sum_{j \in V_{i}} w\left(x_{i}, x_{j}\right) x_{j} \text { with } w\left(x_{i}, x_{j}\right)=\frac{1}{Z_{i}} \exp \left\{-\frac{\left\|N\left(x_{i}\right)-N\left(x_{j}\right)\right\|_{2}^{2}}{h^{2}}\right\}
$$

where $w\left(x_{i}, x_{j}\right)$ is the weight assigned to $x_{j}$ in the reconstruction of $x_{i}$. This weight depends on the similarity between the neighborhood $N\left(x_{i}\right)$ (i.e., 3D patches) of $x_{i}$ and the neighborhood $N\left(x_{j}\right)$ of $\mathrm{x}_{\mathrm{j}}$. The constant $Z_{i}$ ensures that the sum of the weights is equal to 1 . The similarity between patches is estimated within a restricted search volume $V_{i}$ (i.e., a cube volume centered on position $i$ ). As for MRI denoising (Coupe et al., 2008), we observed that using search volume larger than $7 \times 7 \times 7$ voxels only marginally improved results while dramatically increasing computational time. We therefore use neighbourhoods of $7 \times 7 \times 7$ voxels here.

In (Manjon et al., 2010b), it has been shown that such a SR process can be iteratively solved by alternating i) nonlocal means reconstruction and ii) mean consistency correction. In this strategy, the regularization term is first computed using Eq (4). The fidelity term is then applied as a constraint which ensures coherence with the physical model of acquisition: 
$y_{p}-\frac{1}{L} \sum_{i=1}^{L} \hat{x}_{i}=0, \forall p \in y$

In other words, the subsampling consistency (Banerjee and Jawahar, 2008) imposes that the average of the $L$ reconstructed values $\hat{x}_{i}$ must to be equal to the corresponding original value of the LR image (i.e., $H$ is a boxcar). This is done for all the locations $p$ in the LR image by locally adding a corrective offset to the reconstructed values. For example, an upsampling factor of 2 along each axis (i.e., upsampling factor of [2 2 2 2]) forces the average of the $L=8$ reconstructed values to be equal to the corresponding original LR value. As shown in Eq. (1), this constraint is consistent when the noise component is removed. Therefore, the LR image is first denoised with an Rician-adapted denoising filter (Manjon et al., 2012) before performing the reconstruction process. Other point spread functions, i.e., a Gaussian function, could be used for the $H$ operator instead of using a boxcar function (Greenspan, 2009). This would require simply to adapt Eq. (5) by using an appropriately weighted average where the weights would be derived from the considered point spread function.

Finally, this two-step iterative process can be summed up with equations (6) and (7) and is applied until convergence:

$$
\begin{aligned}
& \hat{x}_{i}^{t+1}=\frac{1}{Z_{i}} \sum_{j \in V_{i}} \hat{x}_{j}^{t} \exp \left\{-\frac{\left\|N\left(\hat{x}_{i}^{t}\right)-N\left(\hat{x}_{j}^{t}\right)\right\|_{2}^{2}}{h^{2}}\right\} \\
& \hat{x}^{t+1}=\hat{x}^{t+1}-N N\left(D H \hat{x}^{t+1}-y\right)
\end{aligned}
$$

where $\mathrm{NN}$ is the nearest neighbor interpolation operator to make the size of LR and HR images equal.

As shown in (Coupe et al., 2008) for the nonlocal means method, the filtering parameter $h^{2}$ plays a crucial role. In (Rousseau, 2010), this parameter was fixed to the noise variance. In (Manjon et al., 2010a; Manjon et al., 2010b), a coarse-to-fine approach was proposed where the global value of $h^{2}$ is decreased along the convergence process (i.e., $h=[h / 2, h / 4, h / 8 \ldots]$ ).

In this paper, we propose to improve this later strategy by using a locally adaptive coarse-to-fine framework. In fact, according to the local image content, the optimal speed of convergence can differ (e.g., faster in a constant area than near an edge) and the value of $h^{2}$ should be adapted. Therefore, we propose to use:

$$
\hat{x}_{i}^{t+1}=\frac{1}{Z_{i}} \sum_{j \in V_{i}} \hat{x}_{j}^{t} \exp \left\{-\frac{\left\|N\left(\hat{x}_{i}^{t}\right)-N\left(\hat{x}_{j}^{t}\right)\right\|_{2}^{2}}{2 \sigma_{i}^{2}}\right\}
$$

Where $\sigma_{i}$ is the local variance of $\hat{x}^{0}$ around $x_{i}$ and its value is decreased during convergence $\sigma_{i}=\left[\sigma_{i} / 2, \sigma_{i} / 4, \sigma_{i} / 8 \ldots\right]$. For a given voxel $x_{i}$, the reconstruction is stopped when $\sigma_{i}<\varepsilon$. In this manner, more time is dedicated to reconstruction of edges than to the reconstruction of constant 
areas since in constant areas the initial value of $\sigma_{i}$ is very low. Moreover, slowly decreasing the filtering parameter on the edges limits the introduction of artifacts and prevents the convergence of the reconstruction process to local minima.

\section{- CLASR: Collaborative and locally adaptive super-resolution}

As mentioned above, it is possible to improve SR reconstruction by using HR priors from another image. Originally proposed for the SR reconstruction of LR T2-w data using HR T1-w MRI, we propose to apply this type of approach to DW imaging. Here, we first propose to reconstruct the $b 0$ image using LASR and then to use this reconstructed HR bO image to drive the reconstruction of the individual DW images. By adding $b 0$ image priors, the reconstruction process will be more constrained and thus limit the introduction of artifacts. Therefore, the reconstruction process of a DW image can be rewritten as follows:

$$
\hat{x}_{i, d w}^{t+1}=\frac{1}{Z_{i, d w}} \sum_{j \in V_{i}} \hat{x}_{j, d w}^{t} \exp \left\{-\left(\frac{\left\|N\left(\hat{x}_{i, b 0}^{t}\right)-N\left(\hat{x}_{j, b 0}^{t}\right)\right\|_{2}^{2}}{2 \sigma_{i, b 0}^{2}}+\frac{\left\|N\left(\hat{x}_{i, d w}^{t}\right)-N\left(\hat{x}_{j, d w}^{t}\right)\right\|_{2}^{2}}{2 \sigma_{i, d w}^{2}}\right)\right\}
$$

where $b 0$ and $d w$ indicate the image type. By using Eq (9), higher weights are given to voxels with similar neighborhoods in the $b 0$ image and at the same time with similar neighborhoods in the DW image under study. When a structure is present only in one of either images, the reconstruction process will be driven mainly by the image containing the most suitable information since the weights derived from the other image are non discriminating (i.e., all voxels have similar weights in homogeneous areas). The robustness of this strategy to signal discrepancies in both images has been studied in case of multiple sclerosis lesions, where lesions appear differently in T1w and T2w $\mathrm{MRI}$, and for brain tumor where lesion appears differently in T1w and FLAIR MRI (Manjon et al., 2010a; Rousseau, 2010).

Similarly, in our case, both images will be used during the reconstruction of tissue borders (e.g., edges between white matter and gray matter) since both images share signal properties (see Fig. 1). In white matter, where the signal properties can differ in both images due to the information derived from gradient sensitization, DW images will be used for reconstruction since mainly constant areas will be found in $b 0$ image (i.e., all the weights derived from $b 0$ image will be similar). Finally, when a structure appears in b0 image while corresponding to a constant area in DW image, the reconstruction process will average voxels $x_{j, d w}$ with similar intensities (whatever the weights derived from $b 0$ image are) and thus will preserve the original intensity of the DW image. 


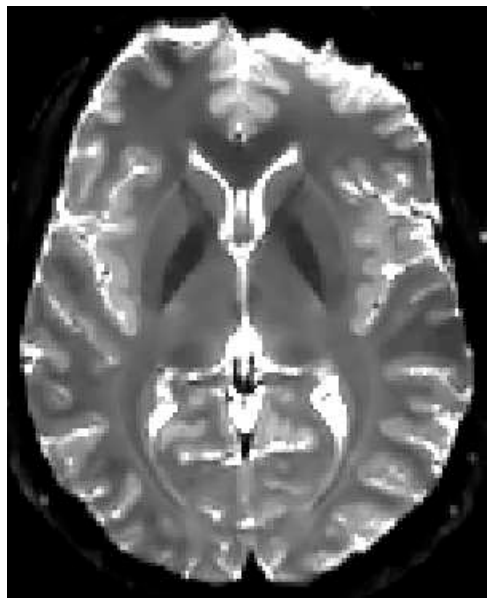

denoised b0 image

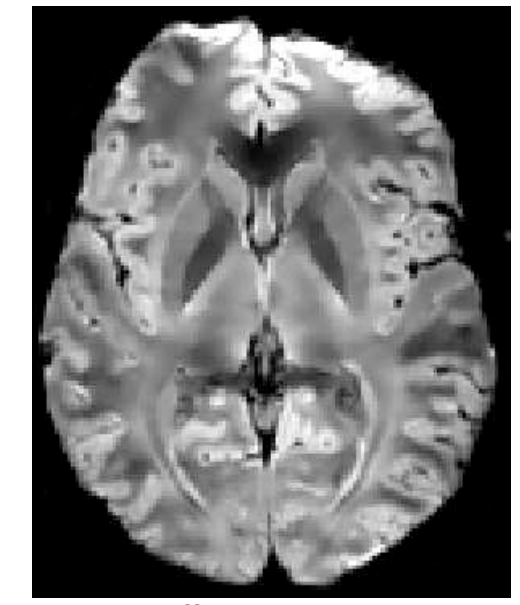

denoised diffusion weighted image

Figure 1: Illustration of the common signal property shared by b0 image and distance weighted images.

In our method, the filtering parameters are automatically and locally adapted. During our experiment, we tried to add a balance parameter to adjust the importance of $b 0$ and DW image as done in (Rousseau, 2010). However, this strategy decreased the image reconstruction quality.

\subsection{Proposed Processing Pipeline}

In order to apply CLASR reconstruction, several pre-processing steps are required. The proposed pipeline consists in the following steps:

- Denoising: As already mentioned, the consistency constraint on mean preservation (see Eq. 5) can be applied only when noise component is removed. Therefore, the first step of our pipeline is to denoise the entire 4D DWI volume using the recently proposed LPCA filter (Manjon et al., 2012). This filter is able to deal with non-stationary noise typically present in DW images and has demonstrated high performance compared to state-of-the-art methods (Tristan-Vega and Aja-Fernandez, 2008; Wiest-Daessle et al., 2008).

- Robust fusion of $b 0$ images: In the case where several $b 0$ images are available, we propose to fuse them using a sharp mean approach (Coupe et al., 2010). This type of fusion strategy limits blurring artifacts inherent to any averaging processes by using a robust estimator.

- LASR reconstruction of b0 image: First, the initialization of b0 image reconstruction is obtained by using trilinear interpolation with the upsampling factor defined by the user

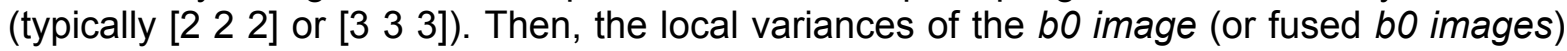
are estimated for the local adaptation of the filtering parameter. Finally, the HR b0 image is reconstructed using Eq. (7) and Eq. (8), iterating until convergence is reached.

- $\quad$ CLASR reconstruction of DW images: The process is similar to LASR reconstruction of $b 0$ image excepted that Eq. (9) is used in place of Eq. (8) where the HR b0 image obtained at the previous step is used as prior. This is done for all the DW images over the 4D DWI volume. 


\subsection{Validation framework}

\section{- Construction of the gold standard}

The advantage of using PBSR compared to interpolation has been widely investigated on synthetic images. Here, we wanted to study the PBSR capabilities in more clinical conditions (i.e., on realistic brain geometry, with real Rician and non-stationary noise, including acquisition artifacts such as patient's motion and eddy current distortion). Therefore, in order to validate the proposed approach, we constructed a gold standard based on a set of in-vivo HR DWI images. To do that, we averaged 10 acquisitions of high resolution DW images in the image space $\left(1.2 \times 1.2 \times 1.2 \mathrm{~mm}^{3}\right)$. First, high resolution DW images were used to ensure that the down-sampling of our gold standard yields a typical clinical DW image resolution (i.e., $2.4 \times 2.4 \times 2.4 \mathrm{~mm}^{3}$ using a factor 2 along each axis). Second, 10 repetitions were averaged to ensure a very high SNR for our gold standard (see Fig. 2). The advantages of performing image processing validation on DW gold standard instead of using synthetic data has been already demonstrated for DW image denoising purposes (Tristan-Vega and Aja-Fernandez, 2009).

DWI datasets were acquired using a 3T MR scanner (Achieva, Philips Medical Systems) equipped with a gradient system providing a maximum gradient strength of $40 \mathrm{mT} / \mathrm{m}$ and with an 8-channel phased array head coil. A standard spin-echo EPI pulse sequence with sensitivity encoding (SENSE) was used to acquire DWI data (b-value: $700 \mathrm{~s} / \mathrm{mm}^{2} ; 21$ diffusion directions) with the following parameters: TE/TR $\sim 60 / 8000 \mathrm{~ms}$; FOV $=215 \times 215 \times 85 \mathrm{~mm}^{2}$; matrix size $=172 \times 172$ with 68 slices and a spatial resolution at $1.2 \times 1.2 \times 1.2 \mathrm{~mm}^{3}$. The acquisition duration for one DWI data set was 5 min $40 \mathrm{~s}$ (see left of Fig 2).

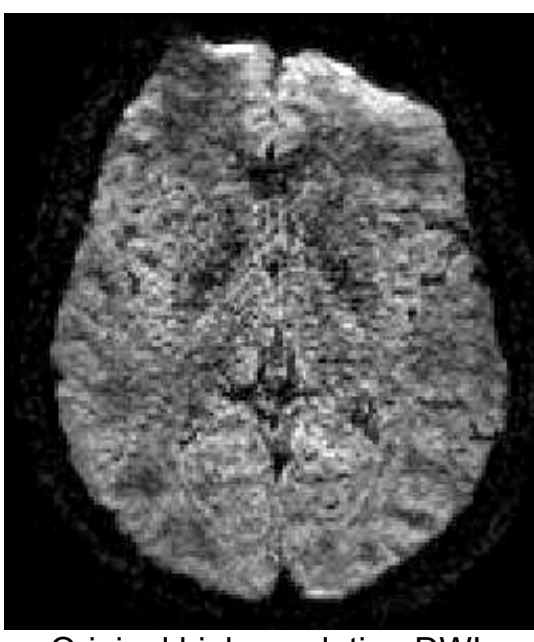

Original high resolution DWI

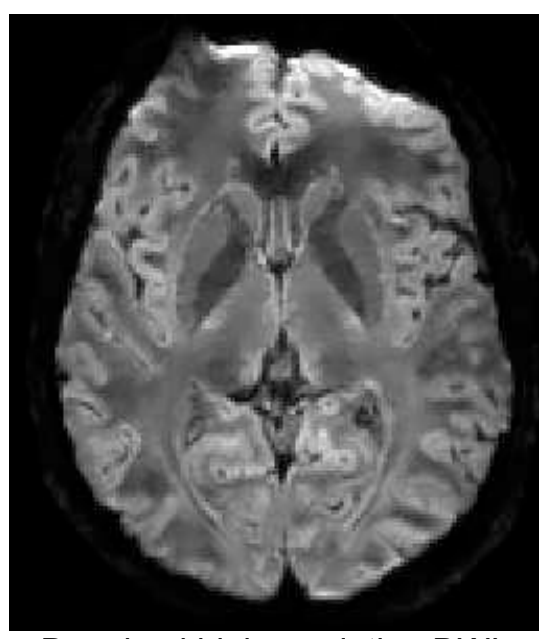

Denoised high resolution DWI

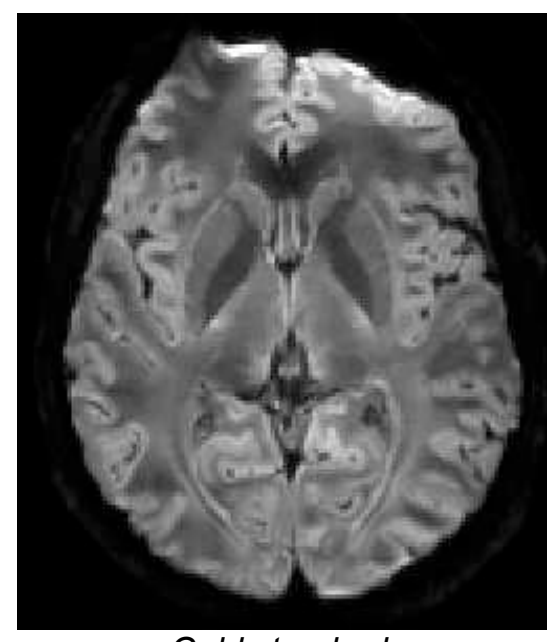

Gold standard

Figure 2: Illustration of the gold standard construction process. Left: one example of the acquired high resolution DWI. Middle: the same image denoised using the LPCA filter (Manjon et al., 2012). Right: the final gold standard obtained by the robust fusion of the 10 denoised high resolution DWI using (Coupe et al., 2010).

Finally, the gold standard construction was achieved by first denoising all the $10 \mathrm{DW}$ images with the LPCA filter (Manjon et al., 2012) (see middle of Fig. 2) and then by fusing the 10 denoised DW images using the robust a mean estimator (Coupe et al., 2010). The LR images used during experiment were simulated by down-sampling our gold standard by a factor 2 using nearest neighbor interpolation along each axis (i.e., [ [ 2 2 2]) what resulted in simulated LR images of $2.4 \times 2.4 \times 2.4 \mathrm{~mm}^{3}$ (see figure 4 ). 
To evaluate the quality of image reconstruction, we compared the proposed approach with trilinear interpolation, tricubic interpolation and third order B-spline interpolation. Moreover, to analyze the advantage of LASR compared to previously proposed PBSR, the original upsampling PBSR method described in (Manjon et al., 2010b) was also included in the comparison.

To evaluate the impact of interpolation and super-resolution methods on diffusion parameters and tensor and HARDI reconstructions, we compared CLASR with trilinear and third order B-spline interpolation methods, since these methods have been recently introduced for DW images resolution enhancement in literature. For instance, trilinear interpolation was used in (Tournier et al., 2012) and B-spline was used in (Dyrby et al., 2011; Raffelt et al., 2012).

\section{- Quality metrics for image reconstruction}

To evaluate the quality of reconstruction two different metrics were used, the usual Peak Signal-toNoise Ratio (PSNR) and the Structural SIMilarity (SSIM) index (Wang et al., 2004). These quality measures were estimated between the reconstructed HR images and the gold standard within a brain mask. The brain mask was estimated first with BET (Smith, 2002) on the b0 image of the gold standard. Due to bad quality of brain extraction, it was manually corrected using ITK-SNAP (Yushkevich et al., 2006). Then, this 3D mask was applied to each DW image to compute PSNR and SSIM.

- Comparison of diffusion parameters

To compare the Fractional Anisotropy (FA) and generalized FA (GFA) obtained with the methods compared, we used Root Mean Square Error (RMSE) within the brain mask previously described and for FA values $>0.2$ (i.e., for white matter approximation). The FA maps were computed with FSL as described in (Smith et al., 2004).

- Comparison of tensor estimation and orientation distribution function (ODF) estimation

Tensor and ODF reconstructions were evaluated using angular distributions estimated between reconstructed DW images and the gold standard.

First, from the diffusion tensor field and principal eigenvector orientation computed with FSL, mean and standard deviation (std) angular error were estimated against the gold standard. Diffusion tensors are visualized as ellipsoids.

Then, the field of constant solid angle ODFs (csa-ODF) was estimated using spherical harmonics (SH) order 4 and lambda regularization of 0.006 (Aganj et al., 2010; Tristan-Vega et al., 2010). From this csa-ODF field, the generalized FA (GFA) was computed and compared with gold standard using RMSE. The GFA corresponds to the ratio of the standard deviation of the ODF over its root mean square (Tuch, 2004). ODFs are visualized as a deformed spherical function, i.e. the radius of the sphere is scaled according to its value on the sphere.

Finally, the field of fiber ODFs (fODFs) was estimated using MRtrix (Tournier et al., 2012) with SH order 4 using a single fiber response function estimated from voxels with FA $>0.7$ and other default parameters. Angular errors for the compared methods were estimated on the field of fODF. For 
fODF maxima extraction, a discrete search over 4,000 points on the sphere is used to extract maxima using the find_SH_peaks command in MRtrix. Then, we used an in-house angular error measure on these maxima based on the implementation provided by the HARDI reconstruction ISBI challenge 2012 (http://hardi.epfl.ch/static/events/2012 ISBI/index.html\#).

\subsection{Implementation details}

All our experiments were performed using MATLAB 7.4 (Mathworks Inc.) on a 6-core Intel(R) Xeon(R) CPU W3670 at $3.2 \mathrm{GHz}$ with $16 \mathrm{~GB}$ RAM. Both LASR and CLASR were implemented using multithreaded C-MEX. Image intensities were scaled linearly between 0-255 and rescaled to original intensity range after processing. The patch size used was $3 \times 3 \times 3$ voxels and the size of search volume $V_{i}$ was $7 \times 7 \times 7$ voxels during all the experiments. The global stopping criterion for convergence was defined as:

$$
\frac{\left|\hat{x}^{t}-\hat{x}^{t-1}\right|}{\left|\hat{x}^{t-1}-\hat{x}^{t-2}\right|} \leq \tau
$$

where $\tau$ was set to 1.2. In addition, for the local stopping criterion $\sigma_{i}<\varepsilon, \varepsilon$ was set to 0.1 . As done for denoising (Coupe et al., 2008), we also used patch pre-selection based on local mean to avoid useless computation. The used condition was defined as:

$$
\left|\mu_{i}-\mu_{j}\right| \leq \delta \sigma_{i}
$$

where $\mu_{i}$ is the mean value of the patch $N_{i}$ and $\delta$ was empirically set to 0.6 since providing good balance between computational time and reconstruction quality. When this condition is not true, the weight $w\left(x_{i}, x_{j}\right)$ is directly set to 0 .

The processing times to reconstruct an HR image at $1.2 \times 1.2 \times 1.2 \mathrm{~mm}^{3}$ from a simulated LR image at $2.4 \times 2.4 \times 2.4 \mathrm{~mm}^{3}$ was approximately 60 seconds with LASR for $b 0$ image and 130 seconds with CLASR for each DW image. However, a number of other optimizations can be performed to reduce the processing time. For example, simply avoiding computations in the background where no useful information is obtained can drastically reduce the computation time. Moreover, GPU implementation of the proposed methods can be achieved, as done for denoising based on nonlocal means (Palhano Xavier de Fontes et al., 2011) to reach near real time computation.

\section{Results}

\subsection{Methods Comparison}

- Quality of Image reconstruction

Table 1 and Figure 3 present PSNR and Mean Structural SIMilarity (MSSIM) obtained by the methods compared. To facilitate comparisons, the improvement in terms of PSNR and MSSIM is also shown using trilinear interpolation as baseline. 
Table 1: Average PSNR and MSSIM estimated between the gold standard and the images reconstructed using the methods compared.

\begin{tabular}{|c|c|c|c|c|c|c|}
\hline & Trilinear & Tricubic & B-spline & Upsampling & LASR & CLASR \\
\hline PSNR in dB & 39.11 & 39.54 & 39.86 & 40.15 & 40.44 & 40.70 \\
\hline MSSIM & 0.9515 & 0.9554 & 0.9578 & 0.9613 & 0.9621 & 0.9649 \\
\hline
\end{tabular}
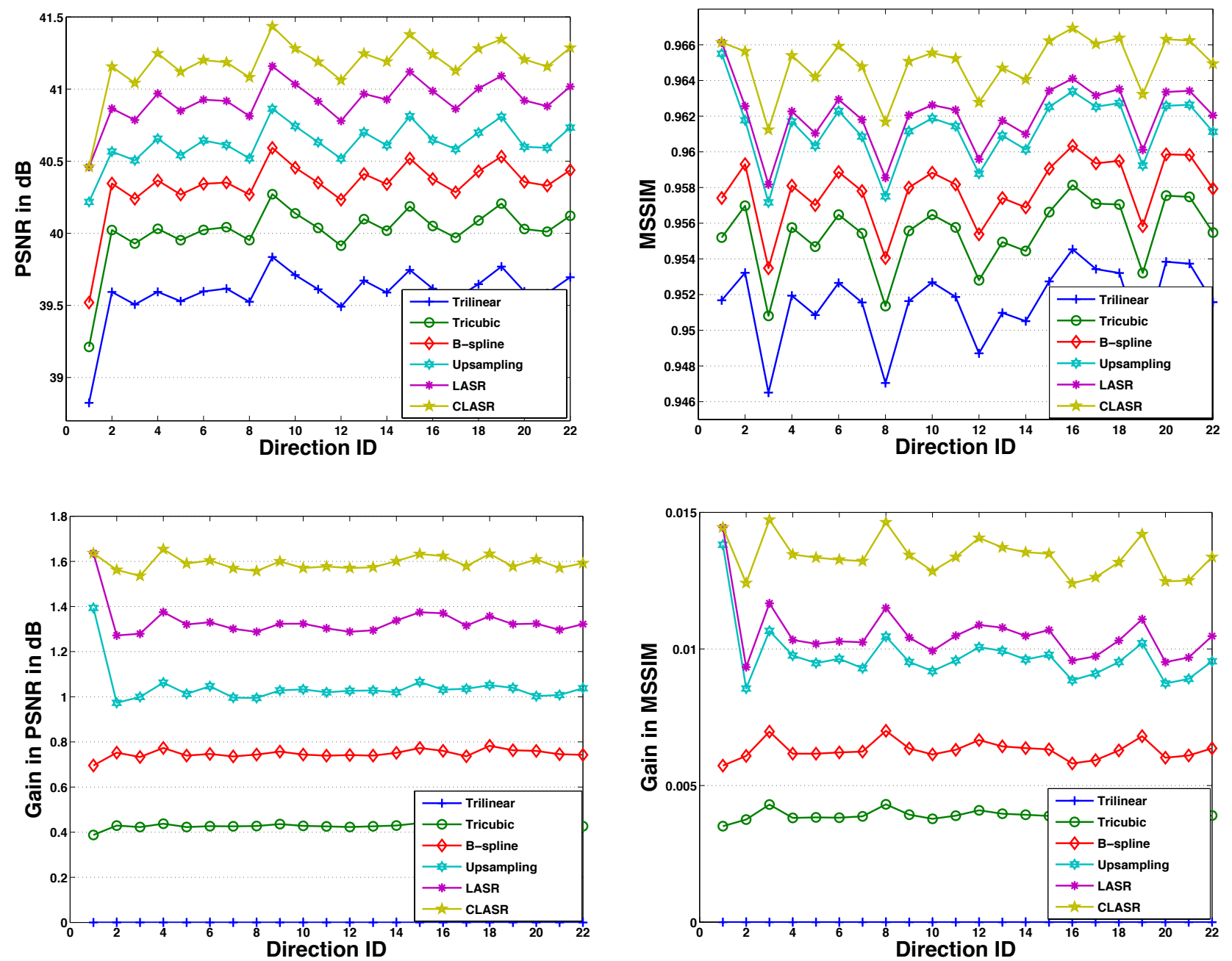

Figure 3: PSNR and MSSIM estimated between the gold standard at $1.2 \times 1.2 \times 1.2 \mathrm{~mm}^{3}$ and the images reconstructed at $1.2 \times 1.2 \times 1.2 \mathrm{~mm}^{3}$ from the simulated LR image at $2.4 \times 2.4 \times 2.4 \mathrm{~mm}^{3}$. Top: plots show the PNSR and MSSIM for the methods compared. Bottom: plots show the improvement in terms of PSNR and MSSIM compared to trilinear interpolation used as baseline.

As already shown on synthetic images in previous studies, PBSR approaches outperform classical interpolation methods. Moreover, B-spline interpolation produced higher PSNR than trilinear and tricubic interpolations. CLASR obtained the highest reconstruction quality in all the studied cases with an average PSNR improvement of $1.6 \mathrm{~dB}$ compared to trilinear interpolation. In addition, CLASR proposed similar improvement for bo image (first point of the series) than for DW images while with LASR the improvement for DW images is lower than the improvement for $b 0$ image. This 
highlights the advantage of using $b 0$ image priors to reconstruct diffusion weighted images. It is also interesting to note that LASR obtained better results than non-adaptive upsampling PBSR method described in (Manjon et al., 2010b). This shows the advantage to locally adapt the filtering parameter compared to global setting.

Figure 4 shows reconstruction of a DW image for trilinear interpolation (used as baseline), for the best interpolation method (B-spline) and the best PBSR method (CLASR). Visually, the reconstruction produced by CLASR is closer to the gold standard. Moreover, CLASR produced less blurred reconstruction with less block-like artifacts compared to usual interpolations. Finally, Bspline interpolation produced images with less block-like artifacts compared to trilinear interpolation.

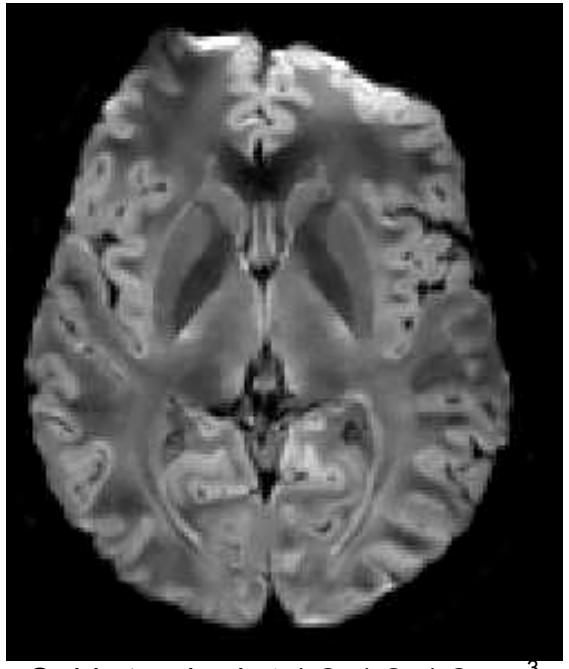

Gold standard at $1.2 \times 1.2 \times 1.2 \mathrm{~mm}$

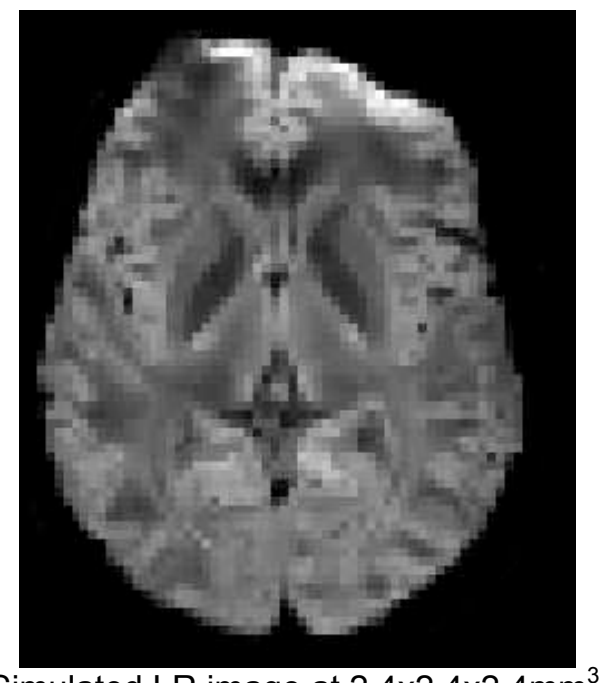

Simulated LR image at $2.4 \times 2.4 \times 2.4 \mathrm{~mm}^{3}$

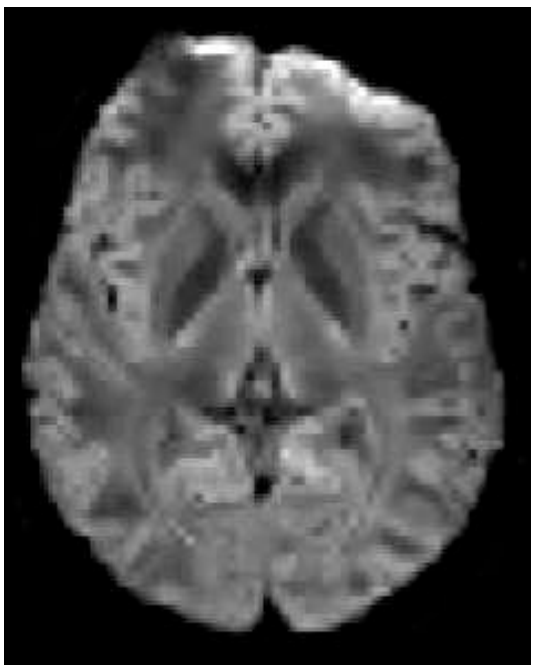

Trilinear interpolation

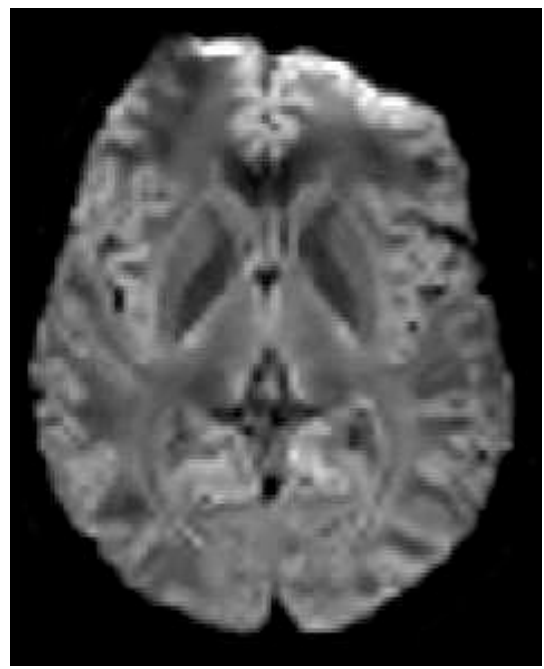

3rd order B-spline interpolation

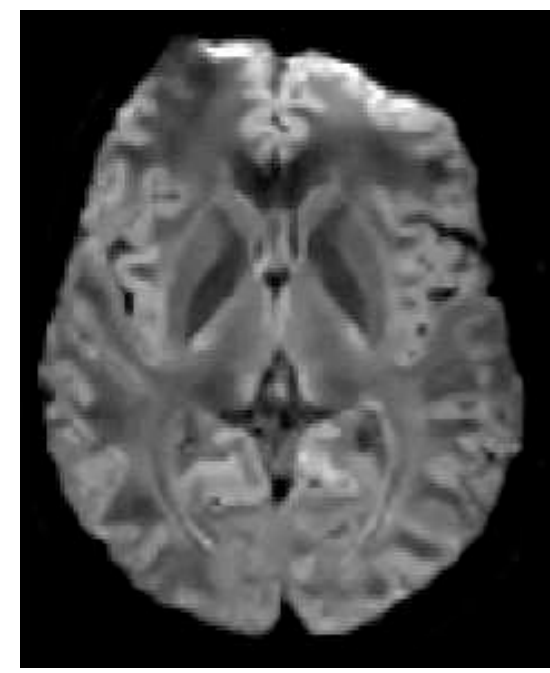

CLASR

Figure 4: Example of diffusion weighted image reconstruction obtained for several methods. Top: The gold standard at $1.2 \times 1.2 \times 1.2 \mathrm{~mm} 3$ and the simulated LR image at $2.4 \times 2.4 \times 2.4 \mathrm{~mm}^{3}$. Bottom: the trilinear reconstruction, the $3^{\text {rd }}$ order $B-s p l i n e$ reconstruction and the results obtained using CLASR. 
To investigate the advantage of using b0 image priors more deeply, we performed two additional experiments. First, we designed an experiment to analyze the role of the high SNR of b0 image (i.e., higher than DW images) in CLASR reconstruction. Second, we proposed an experiment to study the role of the additional image-features provided by b0 image priors compared to DW image priors.

The first experiment consisted in comparing the reconstruction quality of CLASR when using high SNR b0 image and low SNR b0 image. A first dataset was built by adding synthetic Rician noise to the simulated LR image to obtain usual higher SNR for b0 image than for DW images. A second dataset was built by forcing the SNR of b0 image to be equal to the SNR of DWI. The intensity range of b0 image was scaled to the intensity of DWI before adding the same synthetic Rician noise as previously. Both datasets were then processed with the proposed pipeline including the denoising step. In addition to CLASR reconstruction, LASR reconstruction was achieved on both datasets to be used as baseline in order to remove differences resulting from denoising step. Figure 5 shows the results of the experiment in terms of PSNR and MSSIM improvements compared to LASR method. As expected, when using b0 image with lower SNR as priors, the quality of reconstruction decreased since the denoised b0 image had lower image quality. More interestingly, the CLASR reconstruction using low SNR b0 image still outperformed LASR reconstruction. It seems that in addition to the advantage of the higher SNR of b0 image compared to DW image, b0 image priors also provide useful additional image features that help reconstructing DW images.
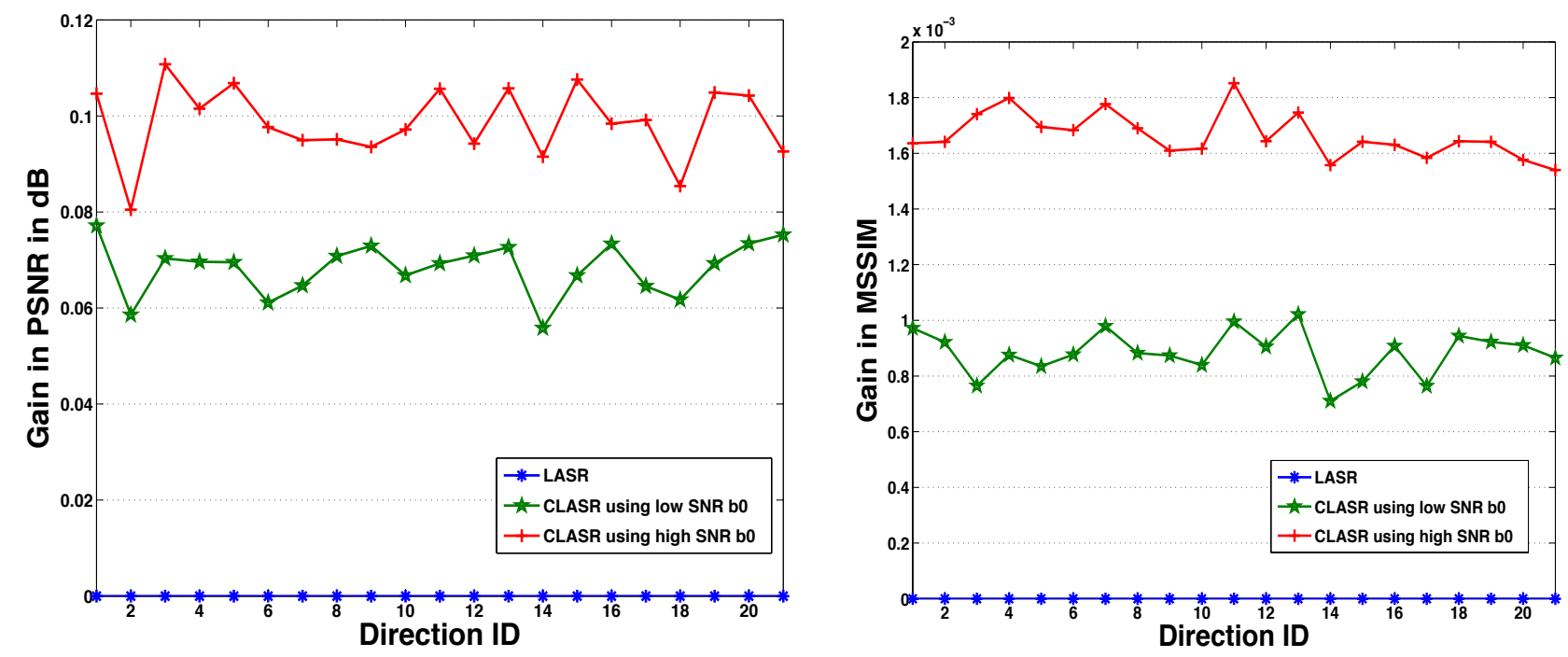

Figure 5: Impact of the SNR of b0 image on CLASR reconstruction quality. Plots show the improvement in terms of PSNR and MSSIM compared to LASR method used as baseline.

In a second experiment, we compared the CLASR reconstruction using b0 image as a prior and the first DW image as a prior. The DW image used as prior was removed from datasets of DW images to be reconstructed. Figure 6 shows the results of the experiment in terms of PSNR and MSSIM improvements compared to LASR method for both datasets. By using b0 image priors better reconstruction quality was obtained. This indicates that b0 image content provides more useful information than DW image content to reconstruct other DW images. This might be attributable to better tissue contrast in b0 image than in DW images (see Figure 1). 

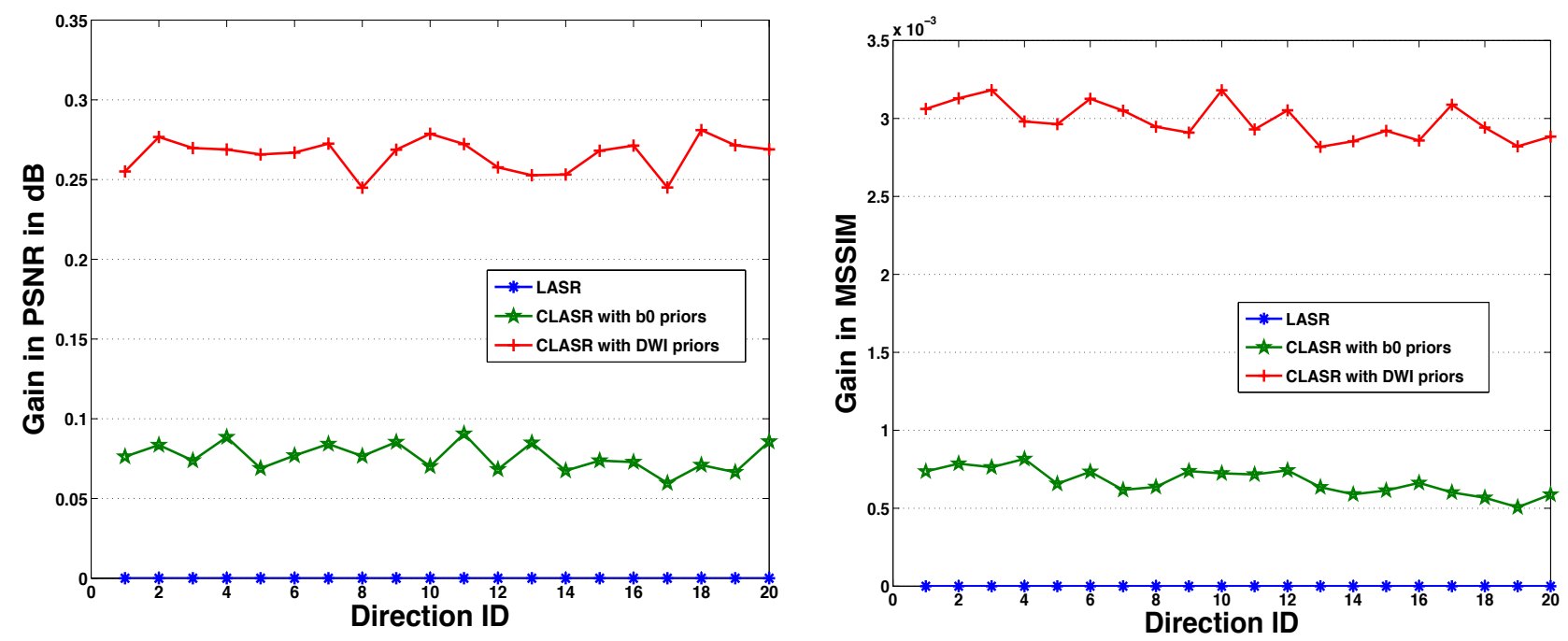

Figure 6: Impact of the choice of priors on CLASR reconstruction quality. Plots show the improvement in terms of PSNR and MSSIM compared to LASR method used as baseline.

\section{- Impact of misalignment on image reconstruction quality}

As mentioned previously, DW datasets can be corrupted by motion artifacts and geometric distortions, making the alignment assumption of b0 and DW images used by CLASR no longer valid. Therefore, we studied the impact of misalignments on the quality of reconstruction obtained with CLASR. First, the displacements between b0 and DW images were obtained with FSL eddy current correction (Smith et al., 2004). The estimated mean displacements in $\mathrm{mm}$ are presented on Figure 7. Then, we analyzed the relationship between image quality in terms of PSNR and the estimated mean displacements. We did not find any significant linear relationship between the quality of CLASR reconstruction and the estimated mean displacements. This result demonstrates the robustness of CLASR to limited misalignments.
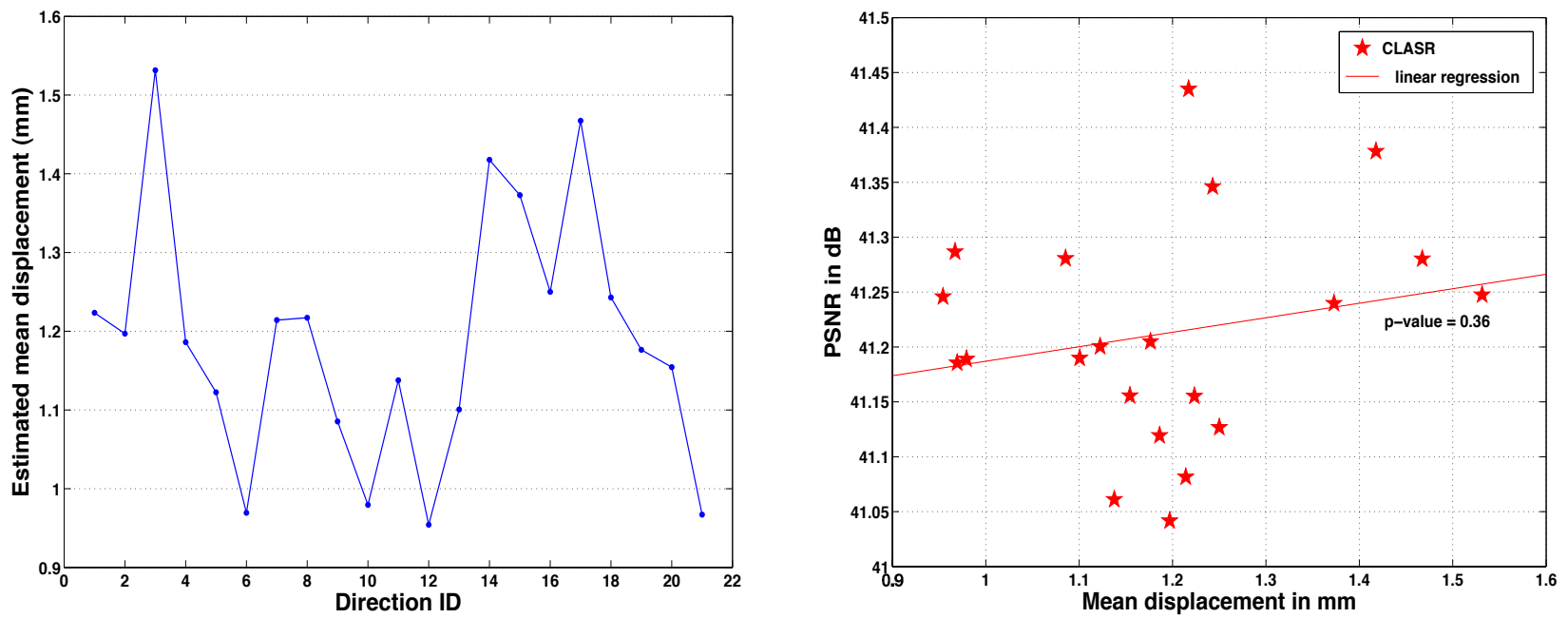

Figure 7: Left: estimated mean displacement in mm using FSL eddy current correction. Right: PSNR obtained with CLASR according to estimated mean displacement. No significant linear relation was found $(p-v a l u e=$ $0.36)$. 
We also studied the impact of performing distortion correction on image reconstruction quality. First, our gold standard was corrected by applying the FSL eddy current correction (see Fig. 8). This correction consists in performing an affine registration of DW images to b0 image and then using trilinear interpolation to match the images. Finally, the first experiment (see Fig. 3) was redone using the obtained corrected gold standard.

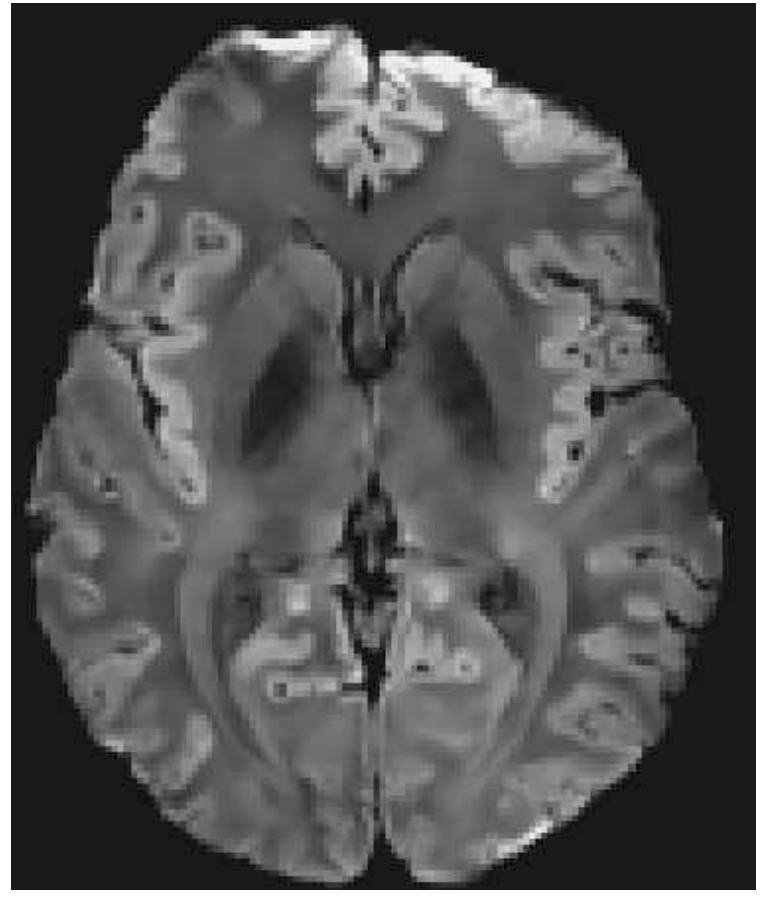

gold standard

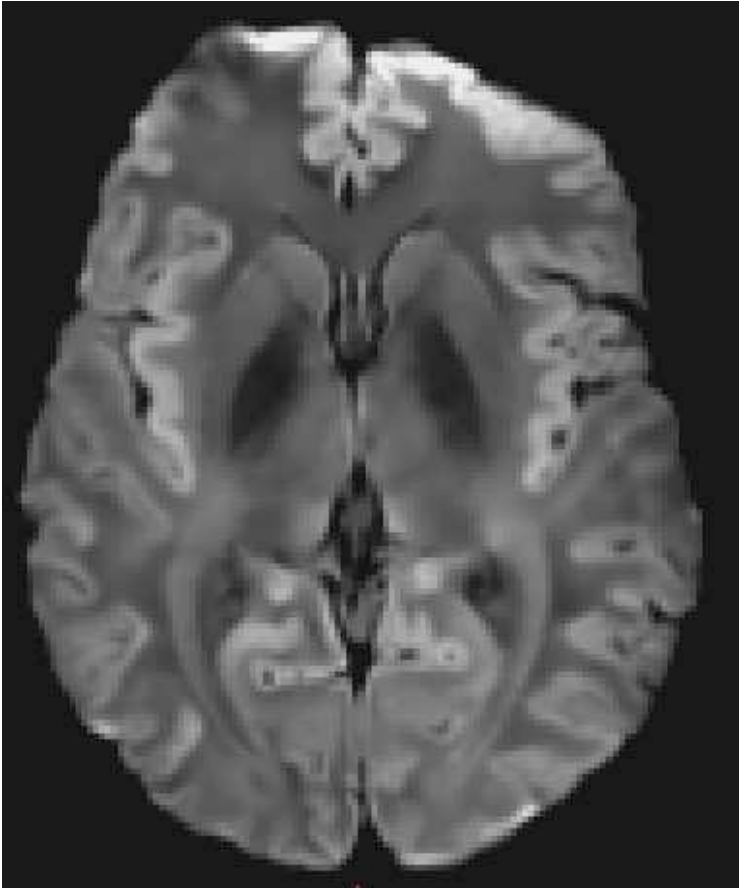

Eddy-current corrected gold standard

Figure 8: Impact of eddy current correction. Left: the gold standard. Right: the eddy-current corrected gold standard suffering from blurring artifacts as compared to the left image.

Figure 9 shows the PSNR and the MSSIM obtained for the methods compared to the corrected gold standard. In all the studied cases, CLASR provided the highest reconstruction quality with an average improvement of $1.9 d B$ in terms of PSNR compared to trilinear interpolation. For the b0 image (i.e., first point of the series) results similar to those presented in Fig. 3 were obtained since b0 image was not impacted by the correction procedure. For DW images, we can observe an increase of PSNR and MSSIM values compared to Fig. 3 for all methods. This is due to the use of a different reference images (i.e., gold standard and corrected gold standard) for quality metric estimation. Therefore, the absolute values of the results obtained for both experiments cannot be compared directly. In addition, we expected a higher advantage of CLASR compared to LASR after registration of DW images onto the b0 image. Although CLASR performed better than LASR, the gain was slightly lower than the gain obtained in the experiment using the uncorrected gold standard. The advantage of better alignment between b0 and DW images was hidden by the use of a blurred gold standard for the global quality metric estimation (see Fig. 8 where the corrected gold standard is blurred everywhere due to trilinear interpolation). In fact, by using corrected gold standard suffering from blurring artifacts, the reconstruction of high frequencies is penalized while it is the main advantage of using SR methods compared to interpolation methods. This can also explain the similar results obtained for upsampling method and B-spline interpolation. 

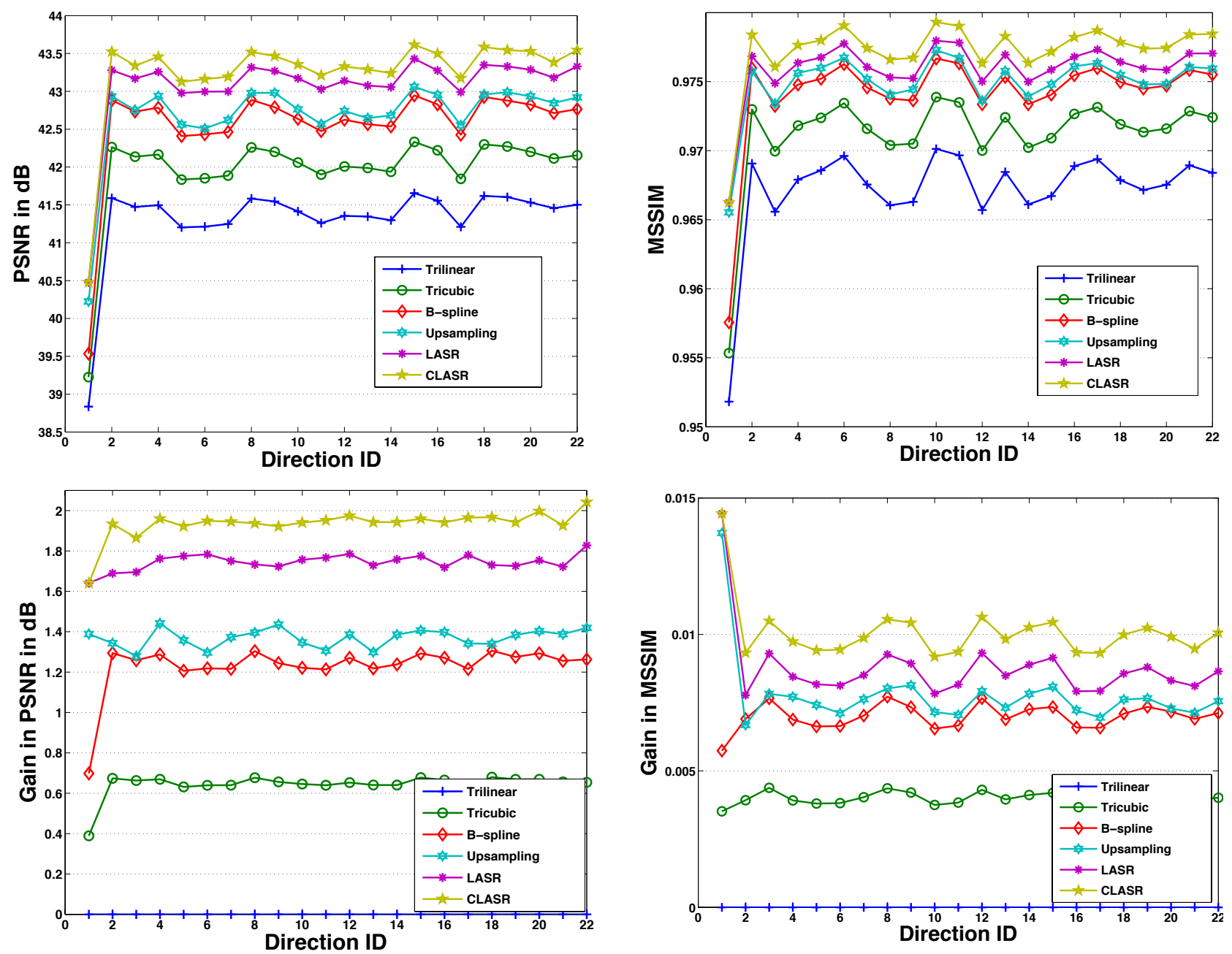

Figure 9: PSNR and MSSIM estimated between the corrected gold standard and the reconstructed images. Top: plots show the PNSR and MSSIM for the method compared. Bottom: plots show the improvement in terms of PSNR and MSSIM compared to trilinear interpolation used as baseline.

\section{- Impact on Fractional Anisotropy (FA)}

Table 2 presents the RMSE of the FA over the white matter for trilinear interpolation, B-spline interpolation and CLASR. CLASR obtained the smallest reconstruction error for this diffusion parameter. As expected from previous results, the reconstructed images with PBSR methods produced FA maps closer to the FA map obtained with the gold standard compared to the interpolation methods. More interestingly, while B-spline interpolation obtained higher PSNR and SSIM than trilinear interpolations for DW image reconstruction (see Figure 3), B-spline interpolation obtained worse results for FA reconstruction. It seems that B-spline interpolation created artifacts that adversely impact FA estimation. Figure 10 shows FA maps estimated from the gold standard, the simulated LR image and the reconstructed DW images using the different methods compared. Visually, the FA map obtained using CLASR is closer to the FA of the gold standard. Finally, the artifacts introduced by B-spline are visible on strong edges such as in the anterior part of the lateral ventricle boundaries. This type of artifact is expected when using high order interpolation methods on quickly varying intensities. As can be noticed in Fig. 10, no such artifacts are created when using the CLASR method. 
Table 2: RMSE of FA estimated over the white matter between the gold standard and the reconstructed images.

\begin{tabular}{|l|l|l|l|}
\hline & Trilinear & B-spline & CLASR \\
\hline RMSE & $7.22 \times 10^{-2}$ & $7.65 \times 10^{-2}$ & $5.73 \times 10^{-2}$ \\
\hline
\end{tabular}

\section{- Impact on Generalized FA, Tensor estimation and ODF estimation}

Table 3 presents the RMSE of the GFA over the white matter between the gold standard and the reconstructed images. The GFA was estimated using constant solid angle ODFs. CLASR obtained the smallest error of reconstruction for this diffusion parameter. Moreover, trilinear interpolation obtained better GFA reconstruction than B-spline interpolation. This result is in line with previous results on FA (see Table 2).

Table 3: RMSE of GFA (generalized fractional anisotropy) estimated over the white matter between the gold standard and the reconstructed images.

\begin{tabular}{|l|l|l|l|}
\hline & Trilinear & B-spline & CLASR \\
\hline RMSE & $1.63 \times 10^{-3}$ & $1.85 \times 10^{-3}$ & $1.12 \times 10^{-3}$ \\
\hline
\end{tabular}

Table 4 presents the angular error using tensor and fiber ODFs models. In all cases studied, CLASR produced the lowest angular error. However, one can note that angular profiles agree closely with the gold standard for all three methods. In the 6-7\% of non-agreement, the underestimation/overestimation is on average equal to 1.0, i.e. there is no strong bias towards underestimation or overestimation of single and crossing configurations. Finally, while for FA and GFA the B-spline interpolant provided the worst results, B-spline interpolation better preserved orientation than trilinear interpolation for the angular study.

Table 4: Angular error in degrees on estimated tensors and fiber ODFs between the gold standard and the reconstructed images. For the ODFs model, the percentage of number of compartments agreement between gold standard and reconstruction techniques is also reported.

\begin{tabular}{|c|c|c|c|c|c|}
\hline \multirow{2}{*}{ Tensor } & \multicolumn{2}{|l|}{ Trilinear } & B-spline & \multicolumn{2}{|l|}{ CLASR } \\
\hline & \multicolumn{2}{|l|}{$1.75 \pm 5.55^{\circ}$} & $1.46 \pm 4.83^{\circ}$ & \multicolumn{2}{|l|}{$1.42 \pm 4.41^{\circ}$} \\
\hline Fiber ODFs & $2.01 \pm 5.05^{\circ}$ & $92.9 \%$ & \begin{tabular}{|l|l}
$1.96+-4.90^{\circ}$ & $93.6 \%$
\end{tabular} & $1.76 \pm 4.55^{\circ}$ & $93.9 \%$ \\
\hline
\end{tabular}




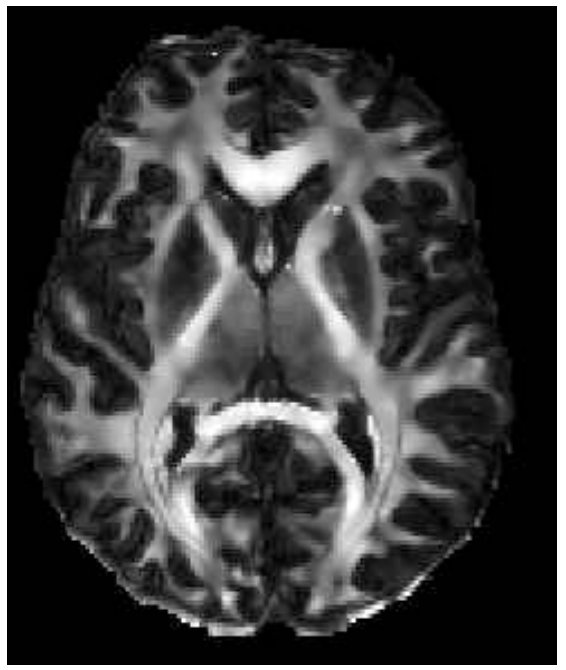

FA map of the gold standard at $1.2 \times 1.2 \times 1.2 \mathrm{~mm}^{3}$

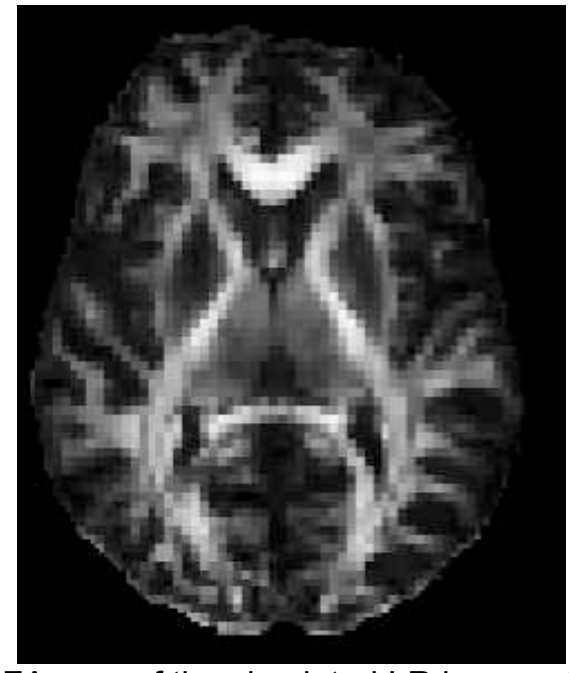

FA map of the simulated LR image at $2.4 \times 2.4 \times 2.4 \mathrm{~mm}^{3}$

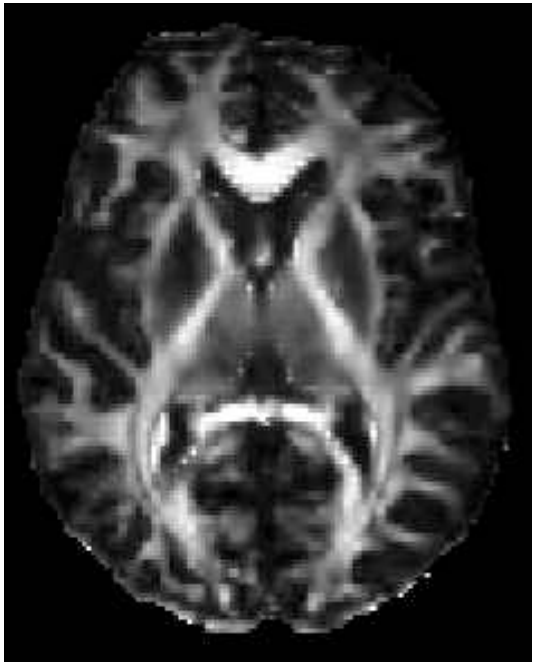

FA map obtained with trilinear interpolation

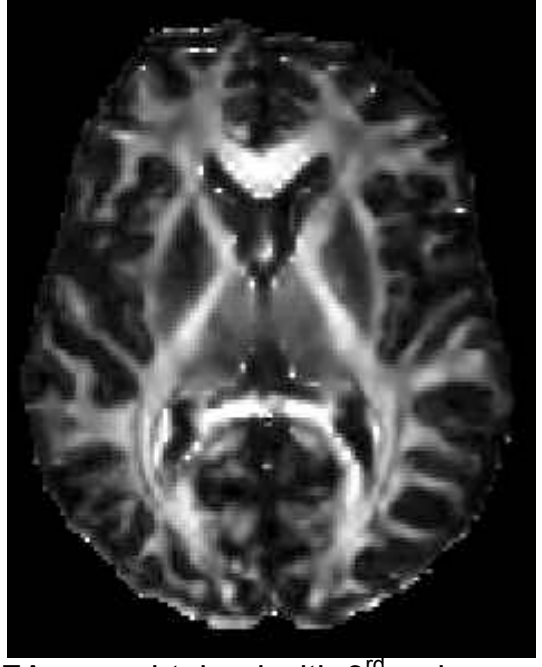

FA map obtained with $3^{\text {rd }}$ order B-spline interpolation

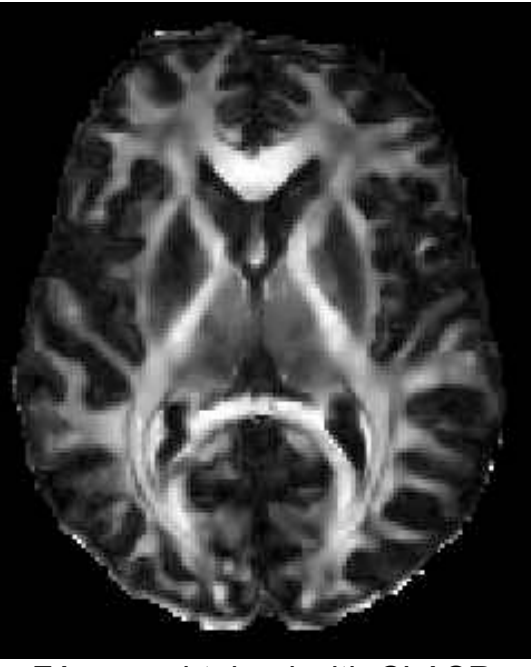

FA map obtained with CLASR

Figure 10: FA maps estimated on the gold standard and the simulated LR image (top). FA maps obtained on reconstructed dataset using trilinear interpolation, $3^{\text {rd }}$ order B-spline interpolation and CLASR (bottom). Visually, the FA map obtained using CLASR is closer to the FA of the gold standard. In addition, B-spline interpolation introduced artifacts visible on strong edges such as near the anterior part of the lateral ventricle boundaries.

Figure 11 presents diffusion tensor estimations around the corpus callosum (CC) for the gold standard and the methods compared. Although all three methods globally preserved tensor orientation, one can note that some single fiber tensors in the CC were degenerated for trilinear and B-spline interpolations while this did not appear with CLASR. In addition, the reconstruction of the boundary between CC and the ventricle is sharper with CLASR.

Figure 12 shows the csa-ODF estimations for the three methods and the gold standard used as reference. As for previous results on the tensor model, one can note that some csa-ODFs in the CC are degenerated for trilinear and B-spline interpolations with shape and principal orientation far from those of the gold standard. In addition, the CC of the trilinear and B-spline interpolations has shrunk considerably on the GFA used as background. This may be due to partial volume effect and contamination of the nearby ventricles. This is reflected in the RMSE on the GFA (see Table 3). 


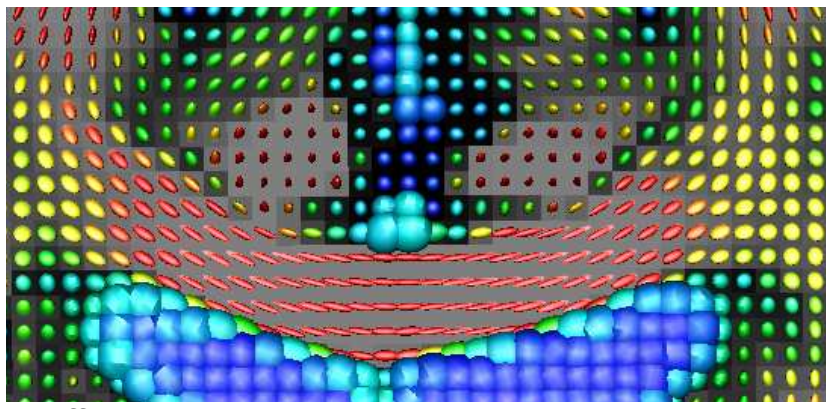

Diffusion Tensor obtained on the gold standard at $1.2 \times 1.2 \times 1.2 \mathrm{~mm}^{3}$

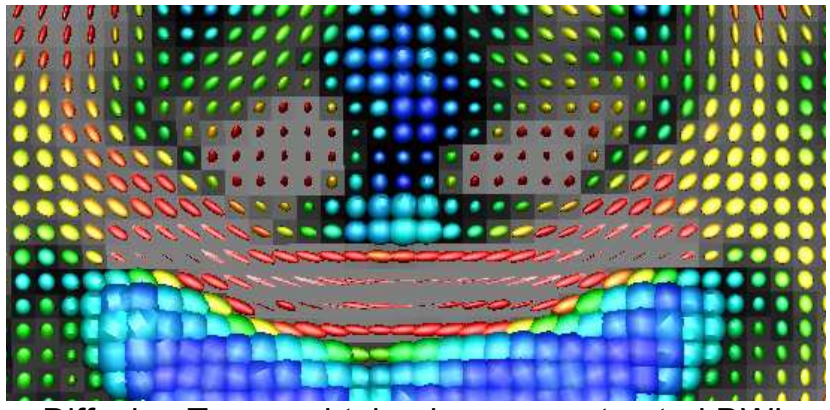

Diffusion Tensor obtained on reconstructed DWI using $3^{\text {rd }}$ order B-spline interpolation

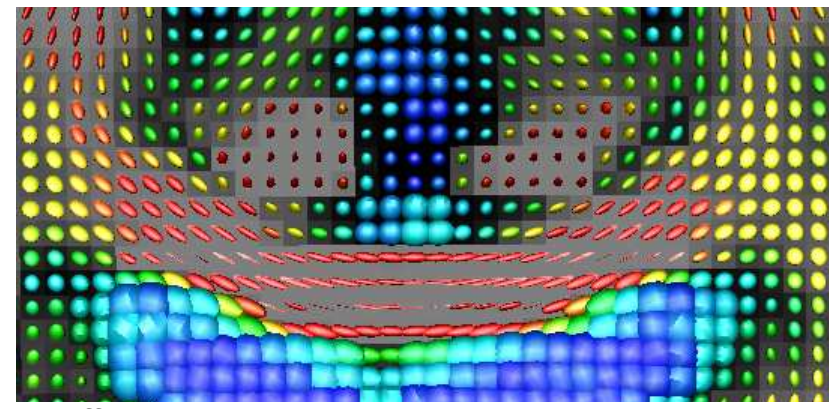

Diffusion Tensor obtained on reconstructed DWI using trilinear interpolation

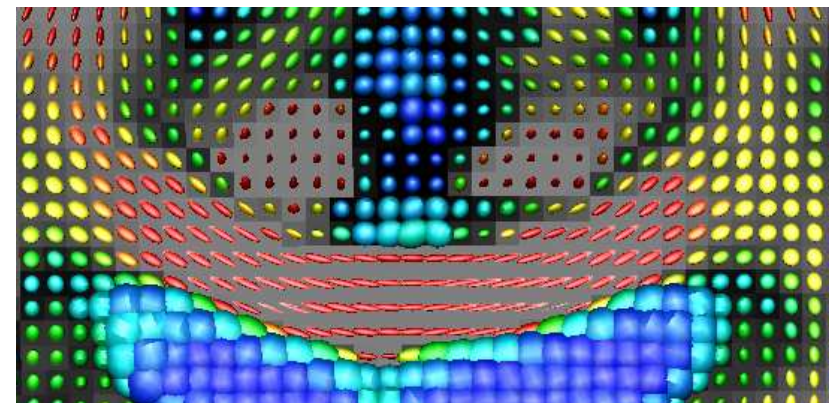

Diffusion Tensor obtained on reconstructed DWI using CLASR

Figure 11: Diffusion tensor estimations on a central coronal slice, centered and zoomed on the corpus callosum (CC). The HR reconstructed images $\left(1.2 \times 1.2 \times 1.2 \mathrm{~mm}^{3}\right)$ were obtained from LR simulated images $\left(2.4 \times 2.4 \times 2.4 \mathrm{~mm}^{3}\right)$ using trilinear interpolation, B-spline interpolation and CLASR. The diffusion tensor estimations obtained on reconstructed images have to be compared with tensors estimated on the gold standard. Some single fiber tensors in the CC are degenerated when using trilinear and B-spline interpolation while this does not appear with CLASR.

\subsection{In-vivo Ultra High Resolution DW images at 3T}

The limited resolution of in-vivo DTI studies (typically, the voxel size of clinical DW images is between $2 \times 2 \times 2 \mathrm{~mm}^{3}$ and $2.5 \times 2.5 \times 2.5 \mathrm{~mm}^{3}$ ) prevents the investigation of finer brain tracts and are subject to PVEs. As already mentioned, acquisition of HR and UHR DW images yields impractical acquisition times for routine clinical studies. This is related to longer acquisition times required to sample the higher frequencies in k-space, but also related to the time dedicated to multiple accumulations required to improve the low SNR of HR images. In this section, we present results of ultra high resolution DW images obtained with CLASR. First, the gold standard (i.e., the average of 10 accumulations) at $1.2 \times 1.2 \times 1.2 \mathrm{~mm}^{3}$ was reconstructed at $0.6 \times 0.6 \times 0.6 \mathrm{~mm}^{3}$ and $0.4 \times 0.4 \times 0.4 \mathrm{~mm}^{3}$. Second, in order to show more practical results from clinical point of view, we also reconstructed ultra-high resolution DW images at $0.6 \times 0.6 \times 0.6 \mathrm{~mm}^{3}$ and $0.4 \times 0.4 \times 0.4 \mathrm{~mm}^{3}$ using a single $\mathrm{HR}$ DWI acquisition requiring 5 minutes 40 seconds of acquisition time.

Figure 13 presents the colored FA maps centered on medial temporal lobe of the gold standard at native resolution (i.e. $1.2 \times 1.2 \times 1.2 \mathrm{~mm}^{3}$ ) and reconstructed at $0.6 \times 0.6 \times 0.6 \mathrm{~mm}^{3}$ and at $0.4 \times 0.4 \times 0.4 \mathrm{~mm}^{3}$ using CLASR. Visually, more details appear on FA estimated on reconstructed images. For instance, fine details of the multi-layered structure of the hippocampus $(\mathrm{HC})$ are better defined after SR. The HC is one of the key structures involved in the early detection of AD (Coupe et al., 2012; Frisoni et al., 2010). In addition, to illustrate the possibility offered by UHR DW images 
in brain structure analysis, we performed streamline deterministic tracking (Tournier et al., 2012) on the field of fiber ODF maxima using the real-time feature of the FiberNavigator (Chamberland et al., 2012). Figure 14 shows a fiber tracking result along the corona radiata obtained on the gold standard. The same seeding region (green box in Fig. 14) in the brainstem was used and the same FA threshold of 0.2 was used as tracking mask in each image. We note a larger extent of the corona radiata $(C R)$ in the reconstructed volume at $0.4 \times 0.4 \times 0.4 \mathrm{~mm}^{3}$ and $0.6 \times 0.6 \times 0.6 \mathrm{~mm}^{3}$ when compared to the original gold standard volume at $1.2 \times 1.2 \times 1.2 \mathrm{~mm}^{3}$. By improving the image resolution, the extent of $\mathrm{CR}$ increased as well, demonstrating the interest of using SR on DW images. In this experiment, we limited the reconstruction at $0.4 \times 0.4 \times 0.4 \mathrm{~mm}^{3}$ for practical reasons in order to keep reconstruction time and memory requirement reasonable during fiber tracking.

Figure 15 shows reconstruction results using only the first acquisition of the 10 datasets used for the gold standard construction. First, as expected, by using one HR acquisition, the SNR of the DW dataset was quite low and thus, resulted in a very noisy FA map estimation and fiber tracking result (see Figs 15 and 16). After denoising, better FA map estimation and fiber tracking were obtained. The denoising filter also removed the intensity bias introduced by Rician noise. This is visible on Figure 15 where the intensity range of FA maps used as background changed after denoising. At the end of the pipeline, the reconstruction provided by CLASR reveals fine details not visible on the original noisy HR DWI. Moreover, the multi-layered structure of the $\mathrm{HC}$ seems better defined on the reconstruction at $0.6 \times 0.6 \times 0.6 \mathrm{~mm}^{3}$ and at $0.4 \times 0.4 \times 0.4 \mathrm{~mm}^{3}$ when compared to the denoised image at $1.2 \times 1.2 \times 1.2 \mathrm{~mm}^{3}$. Finally, the combination of the denoising and super-resolution step provided an FA map close to that obtained for the gold standard (see bottom of Figure 13). As expected, the FA map of the gold standard appears more regular than using a single acquisition. These preliminary results of UHR DW images in less than 6 minutes of acquisition time at 3T are very promising. We also performed fiber tracking using a single acquisition as previously done for the gold standard. The same seeding region in the brainstem was used and a FA threshold of 0.2 was used as tracking mask. The previous remarks done for FA (see figure 15) hold for the fiber tracking results. First, the original noisy HR DW image produced very poor fiber tracking quality (top left of Figure 16). Then, the denoised HR DW image obviously provided better results although the extent of CR was still limited (top right of Figure 16). Finally, the fiber tracking based on the reconstructed images resulted in a larger extent of CR. The estimated fibers appear longer and more dense on the reconstructed DW image. The comparison of the quality of the results obtained at $0.6 \times 0.6 \times 0.6 \mathrm{~mm}^{3}$ (bottom left of Figure 16) and at $0.4 \times 0.4 \times 0.4 \mathrm{~mm}^{3}$ (bottom right of Figure 16) is less obvious. At $0.6 \times 0.6 \times 0.6 \mathrm{~mm}^{3}$ the extent of the CR is larger, but at $0.4 \times 0.4 \times 0.4 \mathrm{~mm}^{3}$ it seems to have less false positive fibers. As expected, compared to the results obtained on the gold standard (see Figure 14), the fiber tracking based on a single acquisition seems more noisy but remains qualitatively close. Finally, in order to verify the reproducibility of our fiber tracking results, we performed the same experiment using the second acquisition of our dataset. The results are presented in Figure 17. As for the first acquisition we observed that fiber tracking is improved after denoising and that a larger extent of CR is obtained after SR. However, while for FA estimation, the use of one acquisition seems to produce satisfying results, the use of 2-3 acquisitions might be required for fiber tracking in order to reveal the full potential of the proposed framework. This aspect will be investigated in future work. 


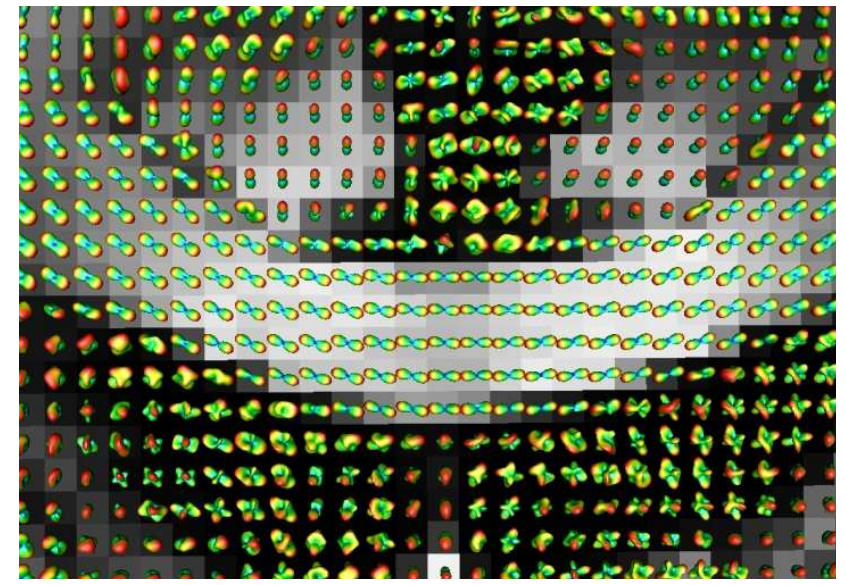

csa-ODF obtained on the gold standard at $1.2 \times 1.2 \times 1.2 \mathrm{~mm}^{3}$

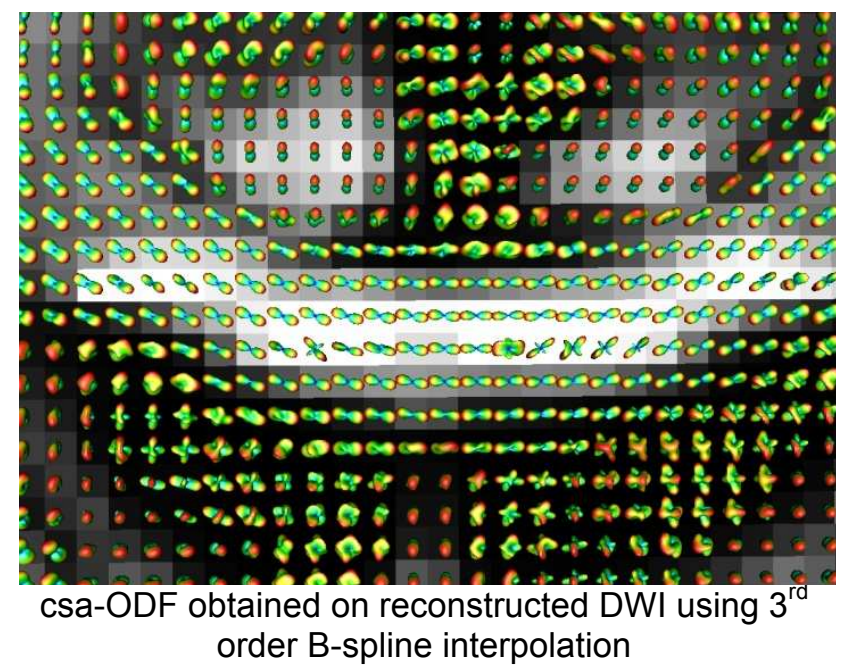

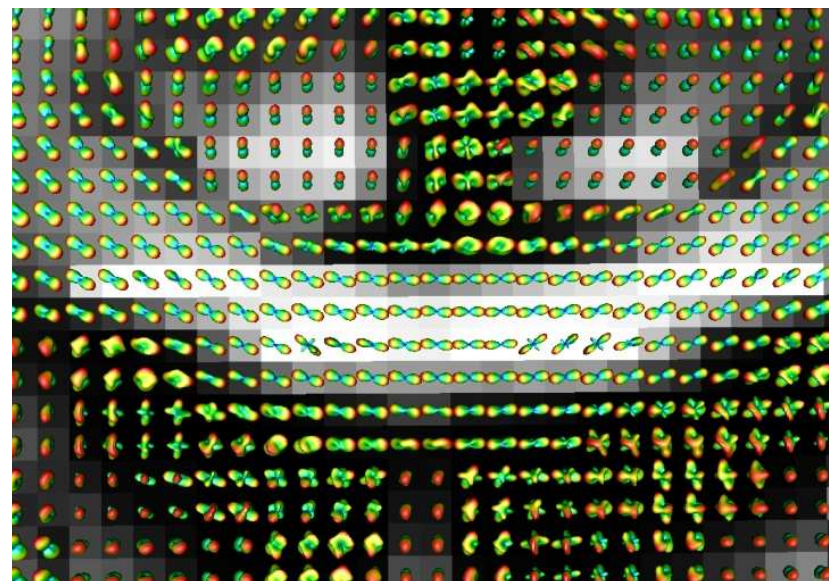

csa-ODF obtained on reconstructed DWI using trilinear interpolation

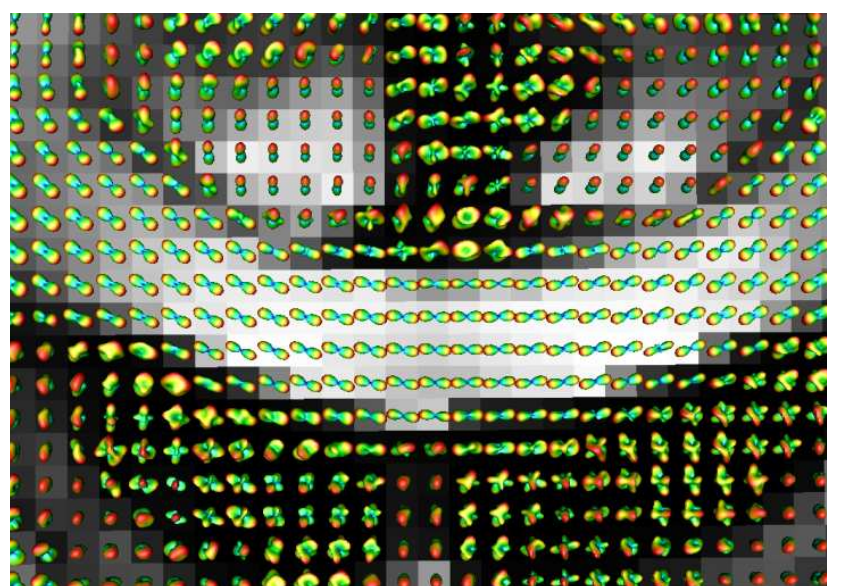

csa-ODF obtained on reconstructed DWI using CLASR

Figure 12: csa-ODF on a central coronal slice, zoomed and centered on the corpus callosum (CC). The HR reconstructed images $\left(1.2 \times 1.2 \times 1.2 \mathrm{~mm}^{3}\right)$ were obtained from LR simulated image $\left(2.4 \times 2.4 \times 2.4 \mathrm{~mm}^{3}\right)$ using trilinear inteprolation, B-spline interpolation and CLASR. The csa-ODF obtained on reconstructed images should be compared with the tensors estimated on the gold standard. Constant solid angle ODF reconstructions were obtained using spherical harmonics of order 4 . These csa-ODFs were used to compute the GFA, which is overlaid underneath the ODF glyphs in this figure. The same intensity range is used for all the GFA maps displayed as background. 


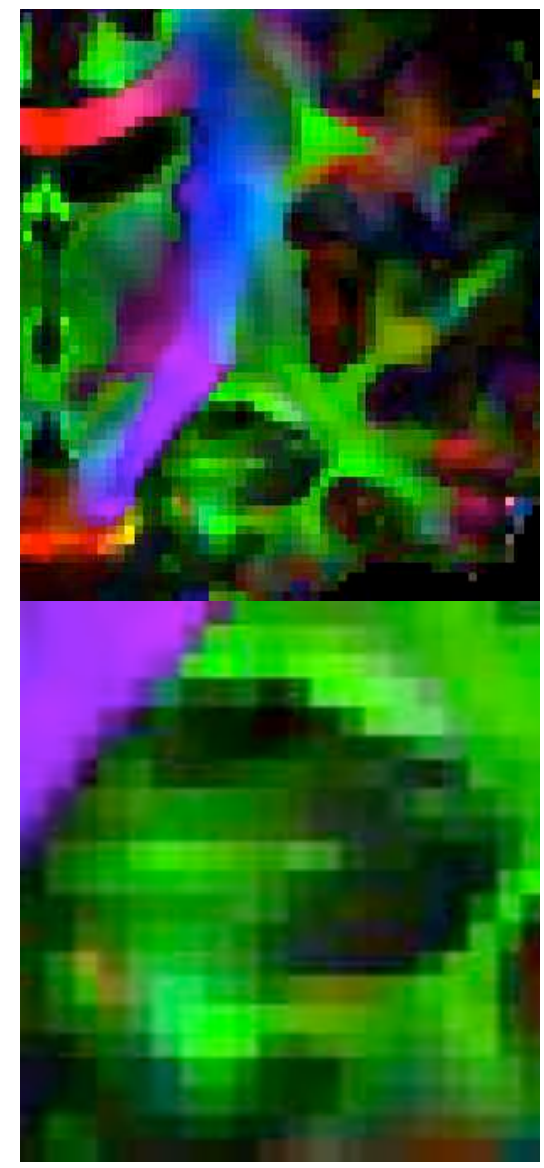

a) Gold standard at $1.2 \times 1.2 \times 1.2$ $\mathrm{mm}^{3}$

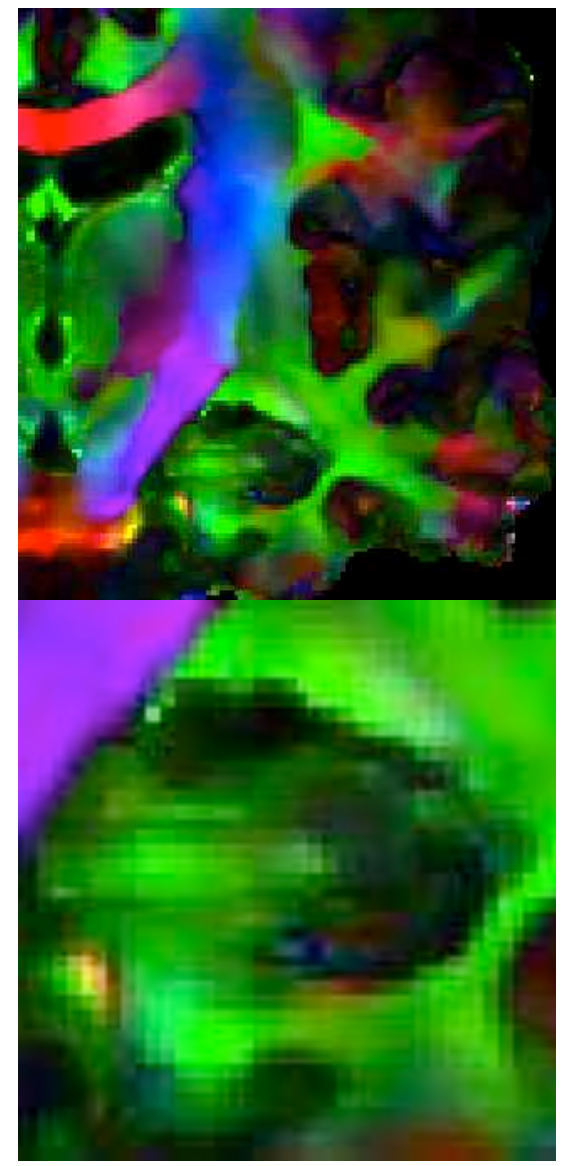

b) Gold standard reconstructed at $0.6 \times 0.6 \times 0.6 \mathrm{~mm}^{3}$ using CLASR

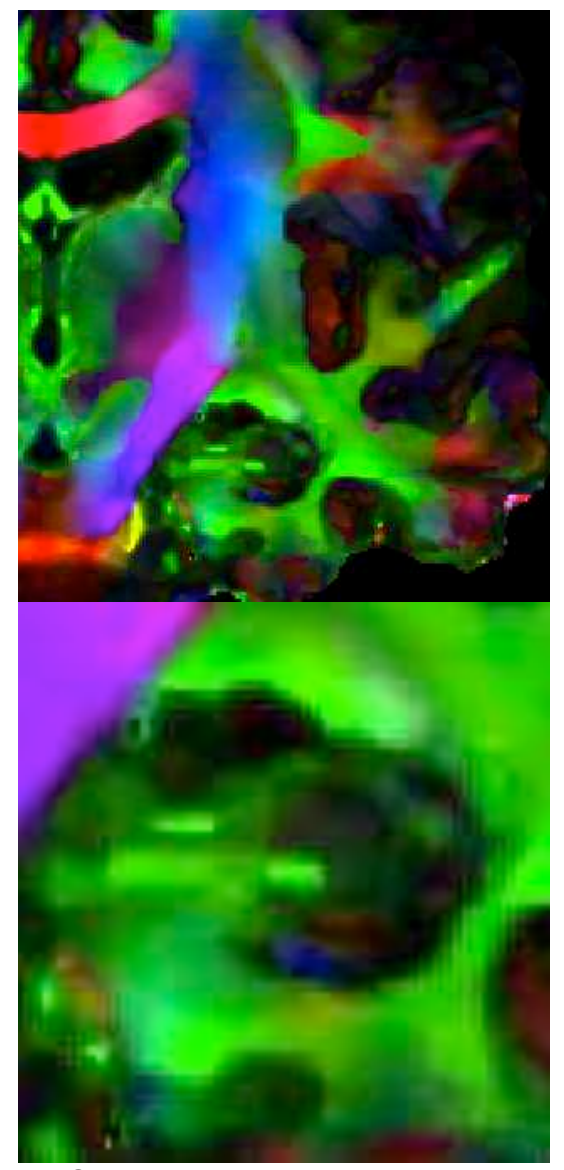

c) Gold standard reconstructed at $0.4 \times 0.4 \times 0.4 \mathrm{~mm}^{3}$ using CLASR

Figure 13: Colored FA maps of a) the gold standard at $1.2 \times 1.2 \times 1.2 \mathrm{~mm}^{3}$ (i.e., robust average of 10 acquisitions), b) the super-resolution of the gold standard at $0.6 \times 0.6 \times 0.6 \mathrm{~mm}^{3}$, and c) the super-resolution of the gold standard at $0.4 \times 0.4 \times 0.4 \mathrm{~mm}^{3}$. The colored FA maps show the medial lobe temporal structures and are centered on the hippocampal area. Fine details of the multi-layered structure of the hippocampus are better defined after super-resolution. 


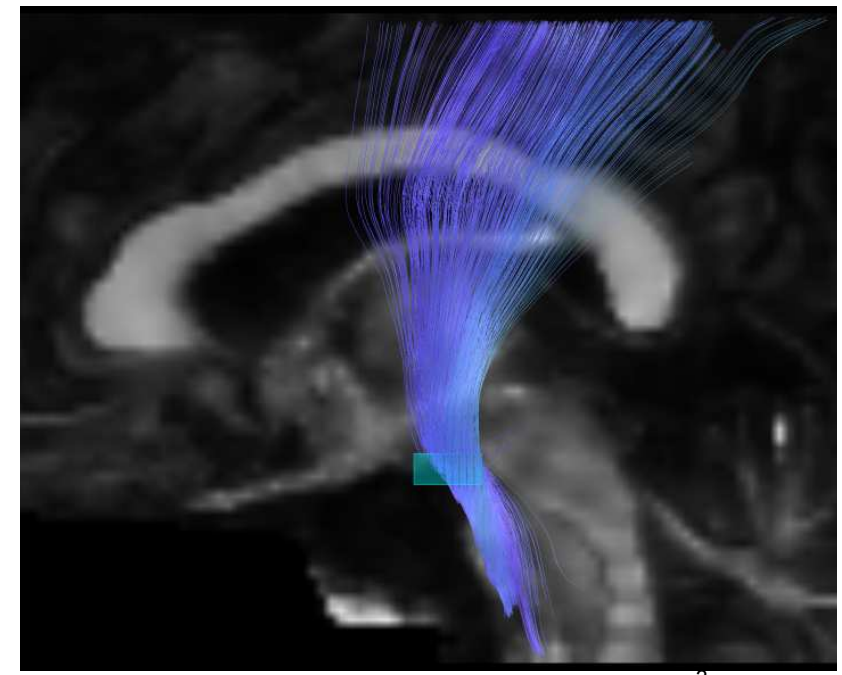

a) Gold standard at $1.2 \times 1.2 \times 1.2 \mathrm{~mm}^{3}$

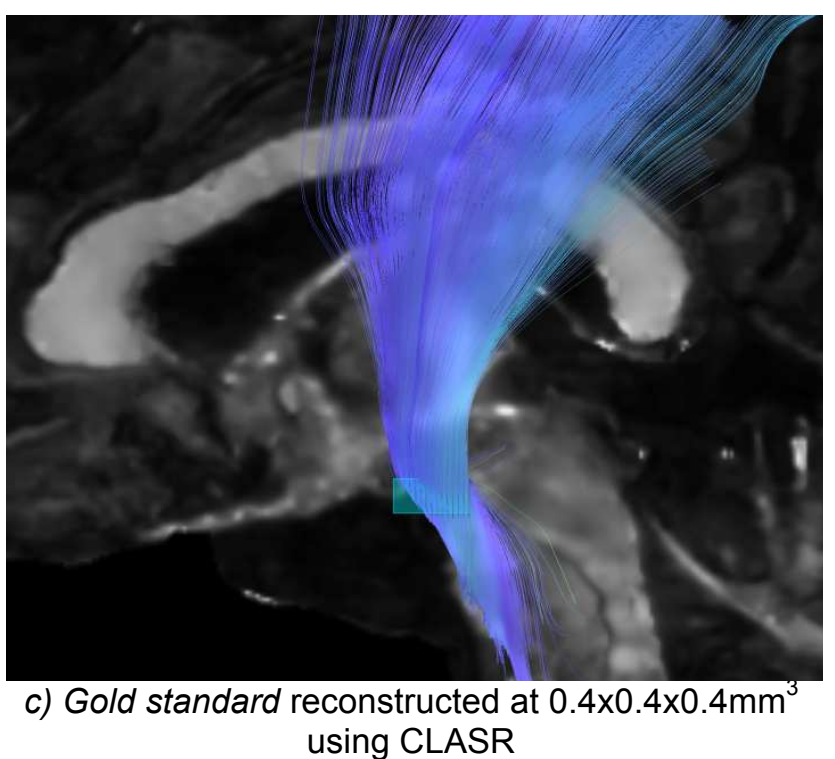

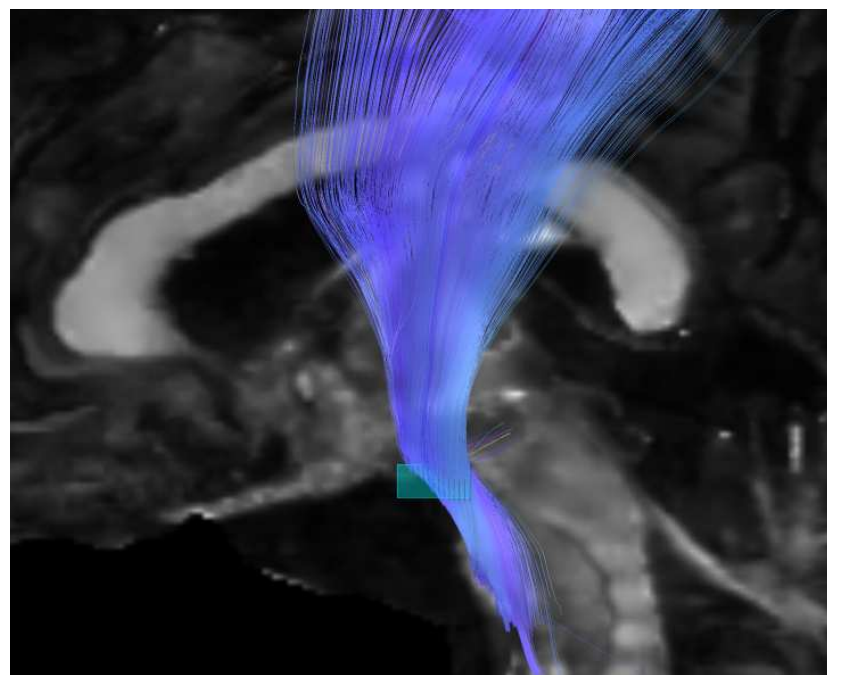

b) Gold standard reconstructed at $0.6 \times 0.6 \times 0.6 \mathrm{~mm}^{3}$ using CLASR

Figure 14: Deterministic fiber ODF tracking on a) the gold standard at $1.2 \times 1.2 \times 1.2 \mathrm{~mm} 3$, b) the superresolution of the gold standard at $0.6 \times 0.6 \times 0.6 \mathrm{~mm}^{3}$ and $\mathrm{c}$ ) the super-resolution of the gold standard at $0.4 \times 0.4 \times 0.4 \mathrm{~mm}^{3}$. The same seeding region in the brainstem was used in each image (green box). A FA threshold of 0.2 is used as tracking mask. We note a larger extent of the corona radiata in the reconstructed images at $0.6 \times 0.6 \times 0.6 \mathrm{~mm}^{3}$ and at $0.4 \times 0.4 \times 0.4 \mathrm{~mm}^{3}$ than in the original gold standard at $1.2 \times 1.2 \times 1.2 \mathrm{~mm}^{3}$. 


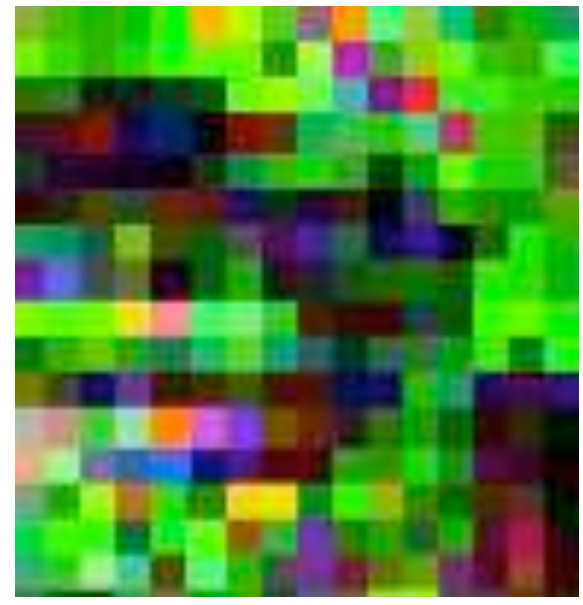

a) First acquisition of HR DWI at $1.2 \times 1.2 \times 1.2 \mathrm{~mm}^{3}$

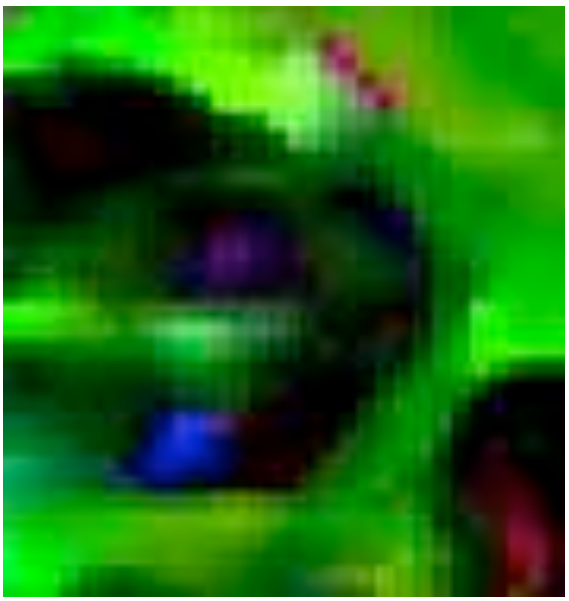

c) Denoised HR DWI reconstructed at $0.6 \times 0.6 \times 0.6 \mathrm{~mm}^{3}$ using CLASR

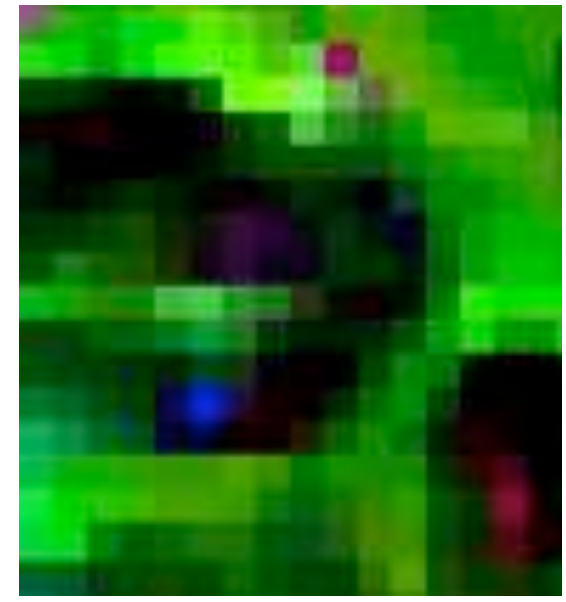

b) Denoised HR DWI at $1.2 \times 1.2 \times 1.2 \mathrm{~mm}^{3}$

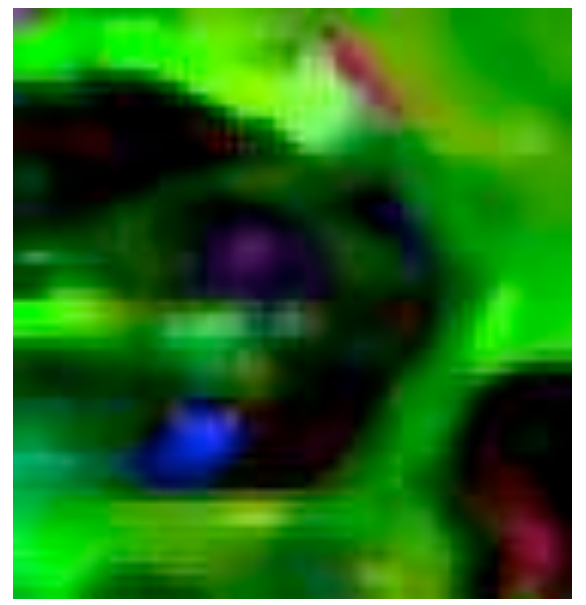

d) Denoised HR DWI reconstructed at $0.4 \times 0.4 \times 0.4 \mathrm{~mm}^{3}$ using CLASR

Figure 15: Coronal zooms on colored FA maps around the hippocampus for a) first acquisition of HR DW image at $1.2 \times 1.2 \times 1.2 \mathrm{~mm}^{3}$, b) the denoised HR DW image using (Manjon et al., 2012), c) the super-resolution of the denoised HR DW image at $0.6 \times 0.6 \times 0.6 \mathrm{~mm}^{3}$ and d) the super-resolution of the denoised HR DW image at $0.4 \times 0.4 \times 0.4 \mathrm{~mm}^{3}$. The combination of the denoising and super-resolution steps provided FA maps close to the FA map obtained for the gold standard (see bottom of Figure 13). 


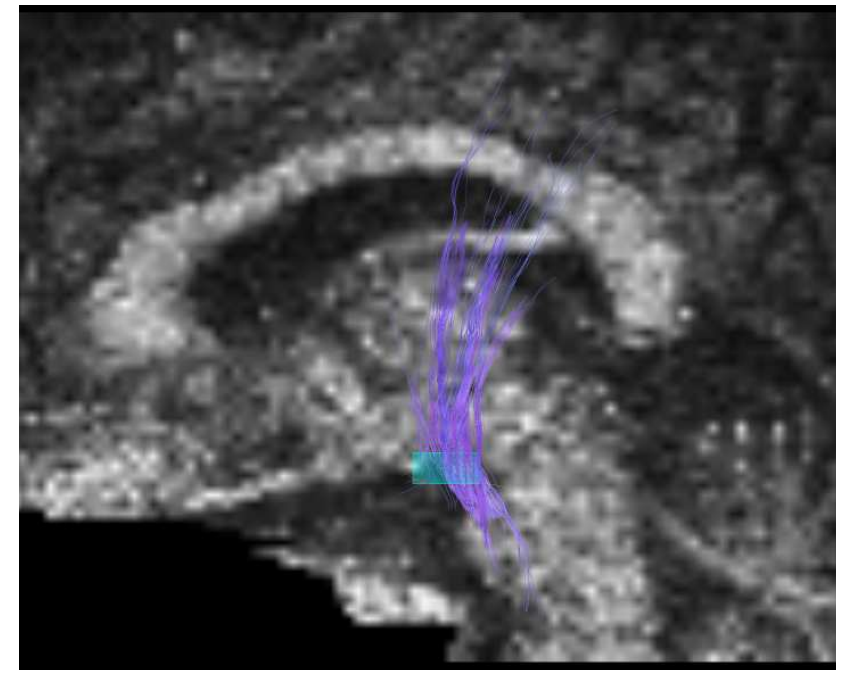

a) First acquisition of the HR DW dataset at $1.2 \times 1.2 \times 1.2 \mathrm{~mm}^{3}$

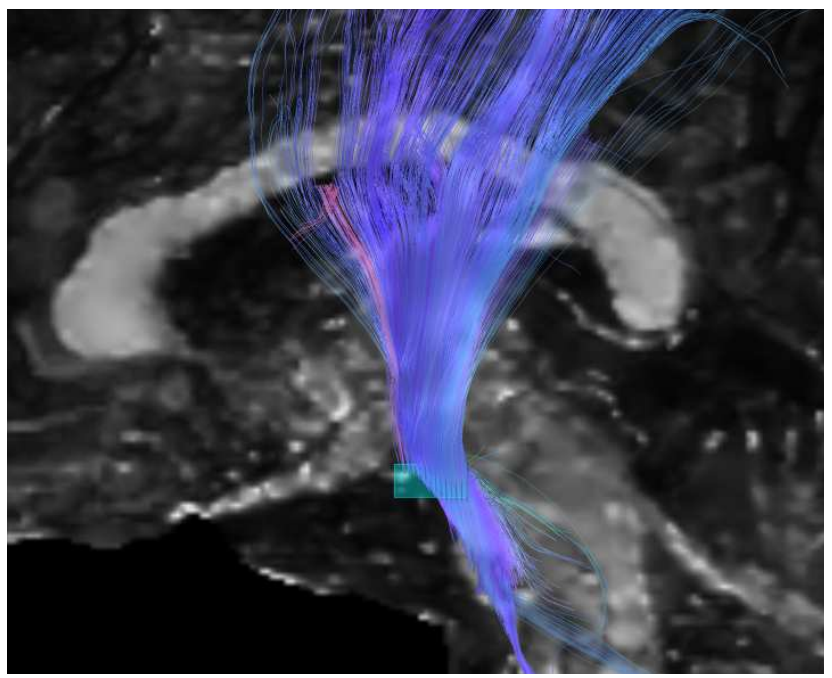

c) Denoised HR DWI reconstructed at $0.6 \times 0.6 \times 0.6 \mathrm{~mm}^{3}$ using CLASR

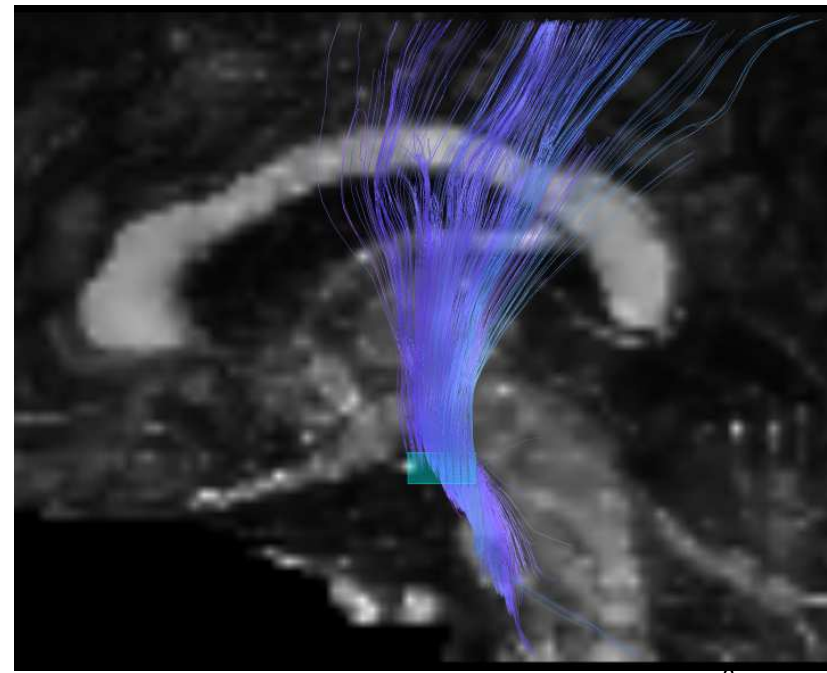

b) Denoised HR DWI at $1.2 \times 1.2 \times 1.2 \mathrm{~mm}^{3}$

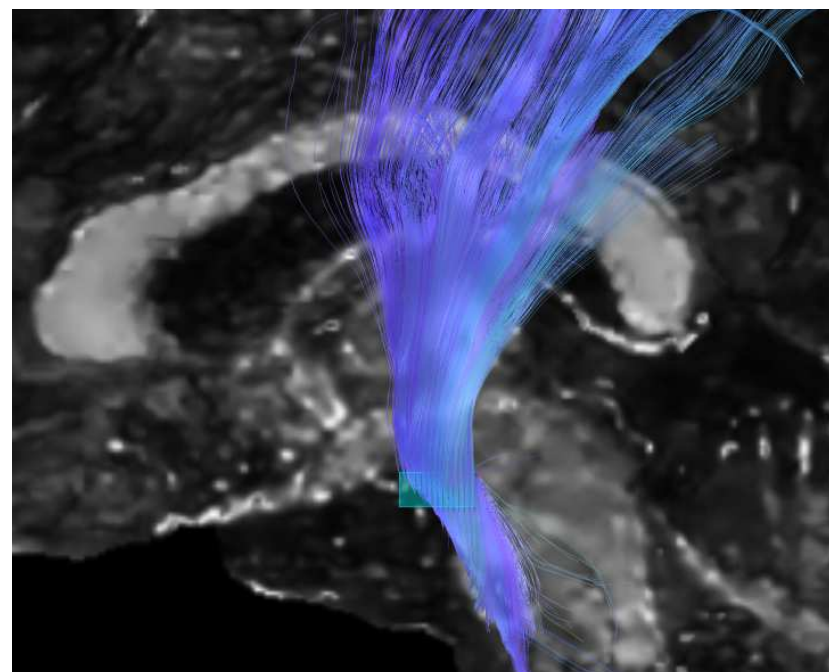

d) Denoised HR DWI reconstructed at $0.4 \times 0.4 \times 0.4 \mathrm{~mm}^{3}$ using CLASR

Figure 16: Deterministic fiber ODF tracking on a) the first acquisition of HR DW image at $1.2 \times 1.2 \times 1.2 \mathrm{~mm}^{3}, \mathrm{~b}$ ) the denoised HR DW image using (Manjon et al., 2012), c) the super-resolution of the denoised HR DW image at $0.6 \times 0.6 \times 0.6 \mathrm{~mm}^{3}$ and d) the super-resolution of the denoised HR DW image at $0.4 \times 0.4 \times 0.4 \mathrm{~mm}^{3}$. The same seeding region in the brainstem was used in each image. A FA threshold of 0.2 was used as tracking mask. The original noisy HR DWI provided poor fiber tracking quality. The denoised HR DW image resulted in better results although the corona radiata (CR) extent was still limited. The use of isotropic reconstructed images at $0.6 \times 0.6 \times 0.6 \mathrm{~mm}^{3}$ and at $0.4 \times 0.4 \times 0.4 \mathrm{~mm}^{3}$ provided a larger extent of the $\mathrm{CR}$. Compared to the results obtained on the gold standard (see Figure 14), the fiber tracking based on a single acquisition seems more noisy but nonetheless qualitatively similar. 


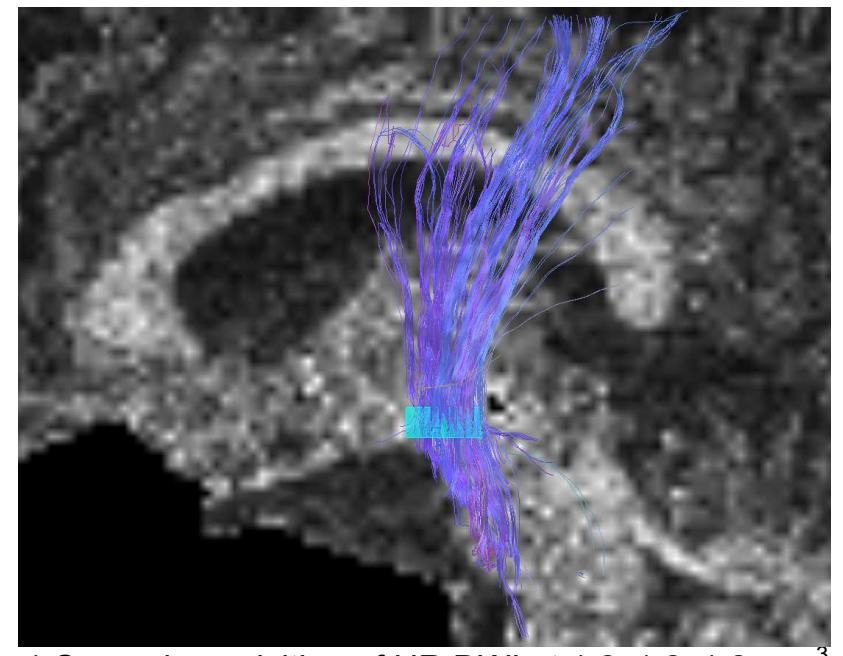

a) Second acquisition of $\mathrm{HR}$ DWI at $1.2 \times 1.2 \times 1.2 \mathrm{~mm}^{3}$

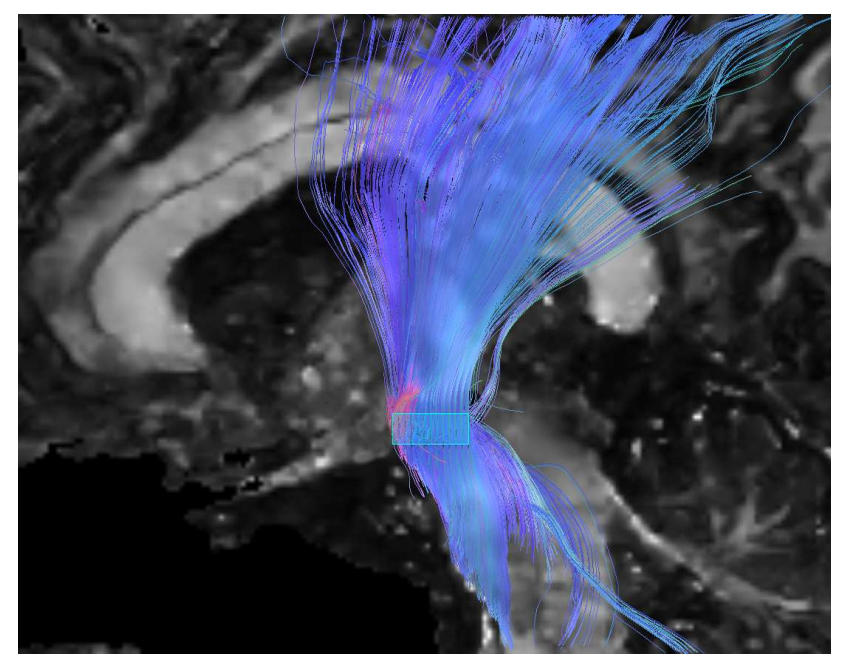

c) Denoised HR DWI reconstructed at $0.6 \times 0.6 \times 0.6 \mathrm{~mm}^{3}$ using CLASR

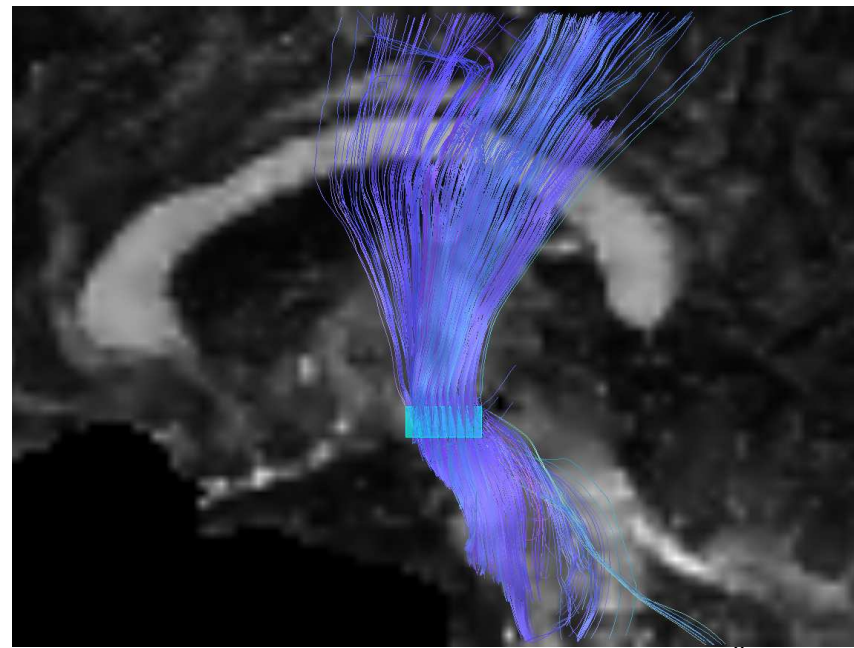

b) Denoised HR DWI at $1.2 \times 1.2 \times 1.2 \mathrm{~mm}^{3}$

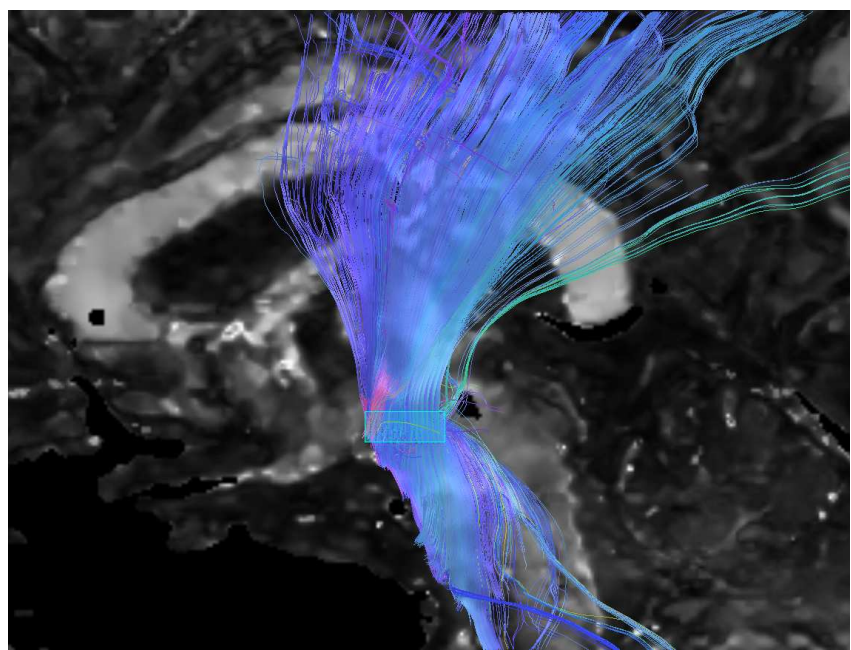

d) Denoised HR DWI reconstructed at $0.4 \times 0.4 \times 0.4 \mathrm{~mm}^{3}$ using CLASR

Figure 17: Deterministic fiber ODF tracking on the second acquisition of HR DW image at $1.2 \times 1.2 \times 1.2 \mathrm{~mm}^{3}$, the denoised HR DW image using (Manjon et al., 2012), the super-resolution of the denoised HR DW image at $0.6 \times 0.6 \times 0.6 \mathrm{~mm}^{3}$ and the super-resolution of the denoised HR DW image at $0.4 \times 0.4 \times 0.4 \mathrm{~mm}^{3}$. The same seeding region in the brainstem was used here and in Fig. 16.

\section{Discussion}

In this study, we investigated the possibility to increase DW image spatial resolution using a new PBSR method. The method described in this paper is an extension and an adaptation to DW image of recently published PBSR methods that are based on the nonlocal means framework. We showed that using the b0 image as a prior, in combination with a locally adaptive reconstruction scheme, improved the reconstruction quality. In recent literature on natural images, new PBSR methods using sparse regularization (Gao et al., 2012; Yang et al., 2010a) seem promising to improve nonlocal super-resolution results even more. Comparison of nonlocal means and sparse-based strategies for MRI denoising demonstrated a slight advantage of the latter. More interestingly, the combination of both provided very competitive results (Manjon et al., 2012) compared to other state-of-the-art methods. In addition, the collaboration of sparse-based methods and redundancybased methods currently provides the best results in image denoising for natural images (Dabov et 
al., 2007). Therefore, we think that this type of collaborative strategy might be an interesting way of investigation for DW image super-resolution.

In this work we studied the impact of the choice of priors on image reconstruction quality. The experiments showed that using high SNR b0 image enables better reconstruction. The advantage of using b0 image is to avoid any additional image acquisitions to save time and thus obviate the need for registration to another modality. In addition, our results suggest that the use of higher quality priors improves reconstruction results. In future work, we will investigate the use of other modalities to drive DW image reconstruction, such as HR T1w and HR T2w images. We think that HR images would provide better priors than the reconstructed HR b0 image using LASR. However, such an approach will require the acquisition of additional HR images and the registration of these images into b0 image space.

The alignment of $b 0$ image and DW image might be corrupted by motion artifacts or/and geometric distortions making Eq. 9 no longer valid. In the case of limited misalignments (e.g, mean displacement inferior to $2 \mathrm{~mm}$ ), we did not observe significant decrease of CLASR reconstruction quality. In addition, it has been shown that multimodal PBSR method still outperforms classical interpolation methods for patient's motion inferior to $4 \mathrm{~mm}$ (Manjon et al., 2010a). In case of large misalignments, the DW dataset will not provide reliable measurement and thus a distortion correction procedure has to be applied first to avoid bias during DW image analysis (Mangin et al., 2002; Rohde et al., 2003), independently of using CLASR. Registration-based approaches usually perform a registration of the corrupted DW image to the b0 image, since it is considered to be less subject to distortions. Evaluation of such methods showed a distortion correction accuracy of approximately $2 \mathrm{~mm}$ (Netsch and van Muiswinkel, 2004). More experiments should be completed for larger distortions. However, this type of analysis is challenging since in case of important misalignments a correction step has to be applied first. We showed that such correction introduces image blurring on the gold standard making the validation results ambiguous. The simulation of realistic nonlinear distortions due to eddy current could be an alternative. However, such realistic simulation remains an open problem. From a practical point of view, we suggest to use CLASR without correction for limited misalignments. For large misalignments, the use of distortion correction cannot be avoided. Our experiments indicated that even after blurring, CLASR still outperforms other methods and thus should be used. Finally, if correction fails while large misalignments are present, LASR could be used instead of CLASR.

In this study, we first validated CLASR by reconstructing $1.2 \times 1.2 \times 1.2 \mathrm{~mm}^{3} \mathrm{DW}$ images from simulated $2.4 \times 2.4 \times 2.4 \mathrm{~mm}^{3} \mathrm{DW}$ images. This situation was close to the reconstruction of clinical DW images (i.e., typically around $2 \times 2 \times 2 \mathrm{~mm}^{3}$ ) at high resolution. During this extensive validation, the quality of image reconstruction for different methods and its impact on diffusion parameters such as FA, GFA, tensor and HARDI estimations were studied. The experiments demonstrated the advantages of using CLASR in all the studied cases. Moreover, we showed that trilinear interpolation was better than B-spline interpolation for FA and GFA estimations, while B-spline interpolation was better than trilinear interpolation for tensor or ODF estimations. In future work, we plan to investigate the interest of using CLASR on standard DW images over a large population. Indeed, the advantage of having DW images with better SNR and better voxel resolution should be analyzed in terms of detection sensitivity using Tract-Based Spatial Statistics (TBSS) analysis (Smith et al., 2006) for instance.

We also investigated the possibility to obtain UHR DW images on a 3T scanner. First, we reconstructed UHR DW image by applying our SR method on HR DW image obtained by averaging multiple acquisitions (i.e., using our gold standard). Second, preliminary results of UHR DW image acquired in a compatible clinical time (i.e., using one acquisition) were presented. We showed that UHR DW images reconstructed with CLASR provided better results than the original HR DW images in both cases. Moreover, for the single acquisition experiment, it appeared that the use of 
efficient denoising steps is crucial. We also analyzed the reproducibility of the experiment using another single acquisition. For studies based on FA map, UHR DW image based on a single acquisition appears promising for a finer analysis of white matter brain structure. However, for studies based on fiber tracking, the use of 2-3 acquisitions might be preferable. Nevertheless, our preliminary results of UHR DW image is encouraging. In future work, we plan to study the interest of using CLASR to analyze fine bundles or difficult crossing areas not easily tractable on usual DW images. In addition, the first qualitative results of fiber tracking proposed in this paper should be more deeply investigated. Although a quantitative analysis of fiber tracking results is very challenging, tools have recently appeared to compare the accuracy and the validity of tracking (Coté et al., 2012; Fillard et al., 2011). Future work should also analyze the impact of the proposed method on fiber tracking algorithms. For instance, fiber ODF reconstruction assumes a deconvolution kernel estimated from the raw data. Therefore, the default parameters to for deconvolution might not be optimal for data that has been denoised and upsampled. The optimal setting of state-of-the-art fiber tracking methods on preprocessed data should be also studied. Nonetheless, we believe that fiber tracking on a properly upsampled data is a promising venue to improve tracking results. In practice, this would enable exploration of fiber bundles near the cortical folds and regions of complex fiber crossings.

\section{Conclusion}

In this paper, we proposed a new single image super-resolution method for DW images. The proposed nonlocal patch-based method uses the b0 image to constrain the reconstruction of diffusion weighted images in order to improve reconstruction. Comparison with traditional interpolation methods such as trilinear and B-spline demonstrated the competitive results of our approach in terms of image reconstruction, FA and GFA reconstructions, and angular reconstruction error for tensor and HARDI models. In addition, first results of ultra high resolution DW image were presented at $0.6 \times 0.6 \times 0.6 \mathrm{~mm}^{3}$ and $0.4 \times 0.4 \times 0.4 \mathrm{~mm}^{3}$ for our gold standard based on 10 acquisitions and for single acquisitions. Finally, fiber tracking results were presented to illustrate the potential of the proposed approach. 


\section{Acknowledgments}

We thank the reviewers for their useful comments that helped improve the paper. We also want to thank the $\operatorname{Pr}$ Louis Collins for proofreading this paper and his fruitful comments. Finally, we want to thank Martine Bordessoules for her help during image acquisition of DWI used to build the phantom. This work has been supported by the French grant "HR-DTI" ANR-10-LABX-57 funded by the TRAIL from the French Agence Nationale de la Recherche within the context of the Investments for the Future program. This work has been also partially supported by the French National Agency for Research (Project MultImAD; ANR-09MNPS-015-01) and by the Spanish grant TIN2011-26727 from Ministerio de Ciencia e Innovación. This work benefited from the use of FSL (http://fsl.fmrib.ox.ac.uk/fsl/fslwiki/), FiberNavigator (code.google.com/p/fibernavigator/), MRtrix software (http://www.brain.org.au/software/mrtrix/) and ITKsnap (www.itk.org). 


\section{References}

Aganj, I., Lenglet, C., Sapiro, G., Yacoub, E., Ugurbil, K., Harel, N., 2010. Reconstruction of the orientation distribution function in single- and multipleshell q-ball imaging within constant solid angle. Magnetic resonance in medicine : official journal of the Society of Magnetic Resonance in Medicine / Society of Magnetic Resonance in Medicine 64, 554-566.

Alexander, A.L., Hasan, K.M., Lazar, M., Tsuruda, J.S., Parker, D.L., 2001. Analysis of partial volume effects in diffusion-tensor MRI. Magnetic resonance in medicine : official journal of the Society of Magnetic Resonance in Medicine / Society of Magnetic Resonance in Medicine 45, 770-780.

Assaf, Y., 2008. Can we use diffusion MRI as a bio-marker of neurodegenerative processes? BioEssays : news and reviews in molecular, cellular and developmental biology 30, 1235-1245.

Bai, Y., Han, X., Prince, J.L., 2004. Super-resolution reconstruction of MR brain images. Proc. of 38th Annual Conference on Information Sciences and Systems (CISS,Äô04).

Banerjee, J., Jawahar, C.V., 2008. Super-Resolution of Text Images Using Edge-Directed Tangent Field. Document Analysis Systems, 2008. DAS '08. The Eighth IAPR International Workshop on, pp. 76-83.

Basser, P.J., Mattiello, J., LeBihan, D., 1994. MR diffusion tensor spectroscopy and imaging. Biophysical journal 66, 259-267.

Buades, A., Coll, B., Morel, J.M., 2005. A non-local algorithm for image denoising. Computer Vision and Pattern Recognition, 2005. CVPR 2005. IEEE Computer Society Conference on, pp. 60-65 vol. 62.

Calamante, F., Oh, S.H., Tournier, J.D., Park, S.Y., Son, Y.D., Chung, J.Y., Chi, J.G., Jackson, G.D., Park, C.W., Kim, Y.B., Connelly, A., Cho, Z.H., 2012a. Super-resolution track-density imaging of thalamic substructures: Comparison with high-resolution anatomical magnetic resonance imaging at 7.0T. Human brain mapping.

Calamante, F., Tournier, J.D., Jackson, G.D., Connelly, A., 2010. Trackdensity imaging (TDI): super-resolution white matter imaging using wholebrain track-density mapping. Neurolmage $53,1233-1243$. 
Calamante, F., Tournier, J.D., Kurniawan, N.D., Yang, Z., Gyengesi, E., Galloway, G.J., Reutens, D.C., Connelly, A., 2012b. Super-resolution trackdensity imaging studies of mouse brain: comparison to histology. Neurolmage 59, 286-296.

Carmi, E., Liu, S., Alon, N., Fiat, A., Fiat, D., 2006. Resolution enhancement in MRI. Magnetic resonance imaging 24, 133-154.

Chamberland, M., Fortin, D., Descoteaux, M., 2012. Real-time fiber tractography : Interactive parameter tuning for neurosurgical interventions., Proceeding of: Human Brain Mapping (HBM), Beijing.

Clouchoux, C., Coupe, P., Manjon, J.V., Guizard, N., Bouyssi-Kobar, M., Lefebre, M., du Plessi, A., Evans, A.C., C, L., 2010. A novel approach for high-resolution image reconstruction for in-vivo fetal brain MRI. Organization for Human Brain Mapping'10.

Coté, M.-A., Boré, A., Girard, G., Houde, J.-C., Descoteaux, M., 2012. Tractometer: Online Evaluation System for Tractography. In: Ayache, N., Delingette, H., Golland, P., Mori, K. (Eds.), Medical Image Computing and Computer-Assisted Intervention ,Äì MICCAI 2012. Springer Berlin Heidelberg, pp. 699-706.

Coupe, P., Eskildsen, S.F., Manjon, J.V., Fonov, V.S., Collins, D.L., 2012. Simultaneous segmentation and grading of anatomical structures for patient's classification: application to Alzheimer's disease. Neuroimage 59, 3736-3747.

Coupe, P., Fonov, V., Manjon, J.V., Collins, D.L., 2010. Template Construction using a Patch-based Robust Estimator. Organization for Human Brain Mapping'10.

Coupe, P., Yger, P., Prima, S., Hellier, P., Kervrann, C., Barillot, C., 2008. An optimized blockwise nonlocal means denoising filter for 3-D magnetic resonance images. IEEE transactions on medical imaging 27, 425-441.

Dabov, K., Foi, A., Katkovnik, V., Egiazarian, K., 2007. Image denoising by sparse 3-D transform-domain collaborative filtering. IEEE transactions on image processing : a publication of the IEEE Signal Processing Society 16, 2080-2095.

Descoteaux, M., Wiest-Daessle, N., Prima, S., Barillot, C., Deriche, R., 2008. Impact of Rician adapted Non-Local Means filtering on HARDI. Medical image computing and computer-assisted intervention : MICCAI ... International 
Conference on Medical Image Computing and Computer-Assisted Intervention 11, 122-130.

Dyrby, T., Lundell, H., Liptrot, M., Burke, W., Ptito, M., Siebner, H., 2011. Interpolation of DWI prior to DTI reconstruction, and its validation. ISMRM.

Fillard, P., Descoteaux, M., Goh, A., Gouttard, S., Jeurissen, B., Malcolm, J., Ramirez-Manzanares, A., Reisert, M., Sakaie, K., Tensaouti, F., Yo, T., Mangin, J.F., Poupon, C., 2011. Quantitative evaluation of 10 tractography algorithms on a realistic diffusion MR phantom. Neurolmage 56, 220-234.

Frisoni, G.B., Fox, N.C., Jack, C.R., Jr., Scheltens, P., Thompson, P.M., 2010. The clinical use of structural MRI in Alzheimer disease. Nature reviews. Neurology 6, 67-77.

Gao, X., Zhang, K., Tao, D., Li, X., 2012. Image super-resolution with sparse neighbor embedding. IEEE transactions on image processing : a publication of the IEEE Signal Processing Society 21, 3194-3205.

Gattellaro, G., Minati, L., Grisoli, M., Mariani, C., Carella, F., Osio, M., Ciceri, E., Albanese, A., Bruzzone, M.G., 2009. White matter involvement in idiopathic Parkinson disease: a diffusion tensor imaging study. AJNR. American journal of neuroradiology 30, 1222-1226.

Gholipour, A., Estroff, J.A., Warfield, S.K., 2010. Robust super-resolution volume reconstruction from slice acquisitions: application to fetal brain MRI. IEEE transactions on medical imaging 29, 1739-1758.

Greenspan, H., 2009. Super-Resolution in Medical Imaging. The Computer Journal 52, 43-63.

Greenspan, H., Oz, G., Kiryati, N., Peled, S., 2002. MRI inter-slice reconstruction using super-resolution. Magnetic resonance imaging 20, 437446.

Hsu, J.L., Van Hecke, W., Bai, C.H., Lee, C.H., Tsai, Y.F., Chiu, H.C., Jaw, F.S., Hsu, C.Y., Leu, J.G., Chen, W.H., Leemans, A., 2010. Microstructural white matter changes in normal aging: a diffusion tensor imaging study with higher-order polynomial regression models. Neurolmage 49, 32-43.

Huang, J., Friedland, R.P., Auchus, A.P., 2007. Diffusion tensor imaging of normal-appearing white matter in mild cognitive impairment and early 
Alzheimer disease: preliminary evidence of axonal degeneration in the temporal lobe. AJNR. American journal of neuroradiology 28, 1943-1948.

Jbabdi, S., Behrens, T.E., Smith, S.M., 2010. Crossing fibres in tract-based spatial statistics. Neurolmage 49, 249-256.

Kindermann, S., Osher, S., Jones, P., 2005. Deblurring and Denoising of Images by Nonlocal Functionals. Multiscale Modeling \& Simulation 4, 10911115.

Lou, Y., Zhang, X., Osher, S., Bertozzi, A., 2010. Image recovery via nonlocal operators. Journal of Scientific Computing 42, 185-197.

Mangin, J.-F., Poupon, C., Clark, C., Le Bihan, D., Bloch, I., 2002. Distortion correction and robust tensor estimation for MR diffusion imaging. Medical Image Analysis 6, 191-198.

Manjon, J.V., Carbonell-Caballero, J., Lull, J.J., Garcia-Marti, G., MartiBonmati, L., Robles, M., 2008. MRI denoising using non-local means. Medical Image Analysis 12, 514-523.

Manjon, J.V., Coupe, P., Buades, A., Collins, D.L., Robles, M., 2010a. MRI superresolution using self-similarity and image priors. International journal of biomedical imaging 2010, 425891.

Manjon, J.V., Coupe, P., Buades, A., Fonov, V., Louis Collins, D., Robles, M., 2010b. Non-local MRI upsampling. Medical Image Analysis 14, 784-792.

Manjon, J.V., Coupe, P., Buades, A., Louis Collins, D., Robles, M., 2012. New methods for MRI denoising based on sparseness and self-similarity. Medical Image Analysis 16, 18-27.

Mignotte, M., 2008. A non-local regularization strategy for image deconvolution. Pattern Recognition Letters 29, 2206-2212.

Miller, K.L., Stagg, C.J., Douaud, G., Jbabdi, S., Smith, S.M., Behrens, T.E., Jenkinson, M., Chance, S.A., Esiri, M.M., Voets, N.L., Jenkinson, N., Aziz, T.Z., Turner, M.R., Johansen-Berg, H., McNab, J.A., 2011. Diffusion imaging of whole, post-mortem human brains on a clinical MRI scanner. Neurolmage $57,167-181$.

Mori, S., van Zijl, P.C.M., 2002. Fiber tracking: principles and strategies - a technical review. NMR in biomedicine 15, 468-480. 
Mukherjee, P., Chung, S.W., Berman, J.I., Hess, C.P., Henry, R.G., 2008. Diffusion Tensor MR Imaging and Fiber Tractography: Technical Considerations. American Journal of Neuroradiology 29, 843-852.

Nedjati-Gilani, S., Alexander, D.C., Parker, G.J.M., 2008. Regularized superresolution for diffusion MRI. Biomedical Imaging: From Nano to Macro, 2008. ISBI 2008. 5th IEEE International Symposium on, pp. 875-878.

Netsch, T., van Muiswinkel, A., 2004. Quantitative evaluation of image-based distortion correction in diffusion tensor imaging. Medical Imaging, IEEE Transactions on $23,789-798$.

Oouchi, H., Yamada, K., Sakai, K., Izu, O., Kubota, T., Ito, H., Nishimura, T., 2007. Diffusion anisotropy measurement of brain white matter is affected by voxel size : Underestimation occurs in areas with crossing fibers. AJNR. American journal of neuroradiology 28, 5 .

Palhano Xavier de Fontes, F., Andrade Barroso, G., Coupé, P., Hellier, P., 2011. Real time ultrasound image denoising. Journal of Real-Time Image Processing 6, 15-22.

Peled, S., Yeshurun, Y., 2001. Superresolution in MRI: application to human white matter fiber tract visualization by diffusion tensor imaging. Magnetic resonance in medicine : official journal of the Society of Magnetic Resonance in Medicine / Society of Magnetic Resonance in Medicine 45, 29-35.

Peyré, G., Bougleux, S., Cohen, L., 2008. Non-local Regularization of Inverse Problems. ECCV 2008 5304, 57-68.

Plenge, E., Poot, D.H.J., Bernsen, M., Kotek, G., Houston, G., Wielopolski, P., van der Weerd, L., Niessen, W.J., Meijering, E., 2012. Super-resolution methods in MRI: Can they improve the trade-off between resolution, signal-tonoise ratio, and acquisition time? Magnetic Resonance in Medicine 68, 19831993.

Poot, D.H., Jeurissen, B., Bastiaensen, Y., Veraart, J., Van Hecke, W., Parizel, P.M., Sijbers, J., 2012. Super-resolution for multislice diffusion tensor imaging. Magnetic resonance in medicine : official journal of the Society of Magnetic Resonance in Medicine / Society of Magnetic Resonance in Medicine.

Poot, D.H., Van Meir, V., Sijbers, J., 2010. General and efficient superresolution method for multi-slice MRI. Medical image computing and 
computer-assisted intervention : MICCAI ... International Conference on Medical Image Computing and Computer-Assisted Intervention 13, 615-622.

Price, S.J., Burnet, N.G., Donovan, T., Green, H.A., Pena, A., Antoun, N.M., Pickard, J.D., Carpenter, T.A., Gillard, J.H., 2003. Diffusion tensor imaging of brain tumours at 3T: a potential tool for assessing white matter tract invasion? Clinical radiology 58, 455-462.

Protter, M., Elad, M., Takeda, H., Milanfar, P., 2009. Generalizing the nonlocal-means to super-resolution reconstruction. IEEE transactions on image processing : a publication of the IEEE Signal Processing Society 18, 36-51.

Raffelt, D., Tournier, J.D., Rose, S., Ridgway, G.R., Henderson, R., Crozier, S., Salvado, O., Connelly, A., 2012. Apparent Fibre Density: a novel measure for the analysis of diffusion-weighted magnetic resonance images. Neurolmage 59, 3976-3994.

Rohde, G., Barnett, A., Basser, P., Marenco, S., Pierpaoli, C., 2003. Comprehensive approach for correction of motion and distortion in diffusion,Äêweighted MRI. Magnetic Resonance in Medicine 51, 103-114.

Rousseau, F., 2008. Brain Hallucination. In: Forsyth, D., Torr, P., Zisserman, A. (Eds.), ECCV 2008. Springer Berlin / Heidelberg, pp. 497-508.

Rousseau, F., 2010. A non-local approach for image super-resolution using intermodality priors. Medical Image Analysis 14, 594-605.

Rousseau, F., Glenn, O.A., Iordanova, B., Rodriguez-Carranza, C., Vigneron, D.B., Barkovich, J.A., Studholme, C., 2006. Registration-based approach for reconstruction of high-resolution in utero fetal MR brain images. Academic radiology $13,1072-1081$.

Sagi, Y., Tavor, I., Hofstetter, S., Tzur-Moryosef, S., Blumenfeld-Katzir, T., Assaf, Y., 2012. Learning in the Fast Lane: New Insights into Neuroplasticity. Neuron 73, 1195-1203.

Scherrer, B., Gholipour, A., Warfield, S.K., 2012. Super-resolution reconstruction to increase the spatial resolution of diffusion weighted images from orthogonal anisotropic acquisitions. Medical Image Analysis.

Smith, S.M., 2002. Fast robust automated brain extraction. Human brain mapping 17, 143-155. 
Smith, S.M., Jenkinson, M., Johansen-Berg, H., Rueckert, D., Nichols, T.E., Mackay, C.E., Watkins, K.E., Ciccarelli, O., Cader, M.Z., Matthews, P.M., Behrens, T.E., 2006. Tract-based spatial statistics: voxelwise analysis of multi-subject diffusion data. Neurolmage 31, 1487-1505.

Smith, S.M., Jenkinson, M., Woolrich, M.W., Beckmann, C.F., Behrens, T.E., Johansen-Berg, H., Bannister, P.R., De Luca, M., Drobnjak, I., Flitney, D.E., Niazy, R.K., Saunders, J., Vickers, J., Zhang, Y., De Stefano, N., Brady, J.M., Matthews, P.M., 2004. Advances in functional and structural MR image analysis and implementation as FSL. Neurolmage 23 Suppl 1, S208-219.

Tournier, J.D., Calamante, F., Connelly, A., 2012. MRtrix: Diffusion tractography in crossing fiber regions. International Journal of Imaging Systems and Technology 22, 53-66.

Tristan-Vega, A., Aja-Fernandez, S., 2008. Joint LMMSE estimation of DWI data for DTI processing. Medical image computing and computer-assisted intervention : MICCAI ... International Conference on Medical Image Computing and Computer-Assisted Intervention 11, 27-34.

Tristan-Vega, A., Aja-Fernandez, S., 2009. Design and construction of a realistic DWI phantom for filtering performance assessment. Medical image computing and computer-assisted intervention : MICCAI ... International Conference on Medical Image Computing and Computer-Assisted Intervention 12, 951-958.

Tristan-Vega, A., Westin, C.F., Aja-Fernandez, S., 2010. A new methodology for the estimation of fiber populations in the white matter of the brain with the Funk-Radon transform. Neurolmage 49, 1301-1315.

Tuch, D.S., 2004. Q-ball imaging. Magnetic resonance in medicine : official journal of the Society of Magnetic Resonance in Medicine / Society of Magnetic Resonance in Medicine 52, 1358-1372.

van Ouwerkerk, J.D., 2006. Image super-resolution survey. Image and Vision Computing 24, 1039-1052.

Van Reeth, E., Tham, I.W.K., Tan, C.H., Poh, C.L., 2012. Super-resolution in magnetic resonance imaging: $A$ review. Concepts in Magnetic Resonance Part A 40A, 306-325.

Wang, Z., Bovik, A.C., Sheikh, H.R., Simoncelli, E.P., 2004. Image quality assessment: from error visibility to structural similarity. IEEE transactions on 
image processing : a publication of the IEEE Signal Processing Society 13, 600-612.

Wiest-Daessle, N., Prima, S., Coupe, P., Morrissey, S.P., Barillot, C., 2008. Rician noise removal by non-Local Means filtering for low signal-to-noise ratio MRI: applications to DT-MRI. Medical image computing and computerassisted intervention : MICCAI ... International Conference on Medical Image Computing and Computer-Assisted Intervention 11, 171-179.

Yang, J., Wright, J., Huang, T., Ma, Y., 2010a. Image Super-Resolution via Sparse Representation. IEEE transactions on image processing : a publication of the IEEE Signal Processing Society.

Yang, J., Wright, J., Huang, T.S., Ma, Y., 2010b. Image super-resolution via sparse representation. Image Processing, IEEE Transactions on 19, 28612873.

Yushkevich, P.A., Piven, J., Hazlett, H.C., Smith, R.G., Ho, S., Gee, J.C., Gerig, G., 2006. User-guided 3D active contour segmentation of anatomical structures: significantly improved efficiency and reliability. Neurolmage 31, 1116-1128.

Zeineh, M.M., Holdsworth, S., Skare, S., Atlas, S.W., Bammer, R., 2012. Ultra-high resolution diffusion tensor imaging of the microscopic pathways of the medial temporal lobe. Neurolmage. 